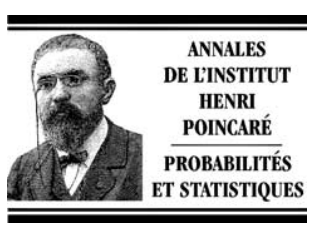

www.elsevier.com/locate/anihpb

\title{
On the sample paths of diagonal Brownian motions on the infinite dimensional torus
}

\author{
A. Bendikov, L. Saloff-Coste ${ }^{1}$ \\ Department of Mathematics, Malott Hall, Cornell University, Ithaca, NY 14853-4201, USA \\ Received 10 October 2002; accepted 26 February 2003
}

\section{Abstract}

We study the intrinsic regularity of the sample paths of certain Brownian motions on the infinite dimensional torus $\mathbb{T}^{\infty}$. Here, intrinsic regularity means regularity with respect to the intrinsic distance $d$ associated to the Brownian motion in question. We prove that some Brownian motions on $\mathbb{T}^{\infty}$ satisfy the classical law of iterated logarithm, that is,

$$
\limsup _{t \rightarrow 0} \frac{d\left(X_{0}, X_{t}\right)}{\sqrt{4 t \log \log (1 / t)}}=1
$$

whereas others do not. For instance, for any slowly varying function $\psi$ such that $\psi(t) \geqslant \log \log t$ at infinity, we give examples of Brownian motions on $\mathbb{T}^{\infty}$ such that

$$
0<\limsup _{t \rightarrow 0} \frac{d\left(X_{0}, X_{t}\right)}{\sqrt{4 t \psi(1 / t)}}<\infty
$$

We prove similar results concerning the uniform modulus of continuity of Brownian paths on the time interval $[0,1]$. Namely, we prove that there are Brownian motions on $\mathbb{T}^{\infty}$ satisfying the classical Lévy-type result but, for any slowly varying function $\psi$ such that $\psi(t) \geqslant \log t$ at infinity, we give examples of Brownian motions on $\mathbb{T}^{\infty}$ such that

$$
0<\lim _{\varepsilon \rightarrow 0} \sup _{\substack{0<s<t \leqslant 1 \\ t-s \leqslant \varepsilon}} \frac{d\left(X_{s}, X_{t}\right)}{\sqrt{4 t \psi(1 / t)}}<\infty .
$$

We also obtain partial results concerning the behavior of

$$
\liminf _{t \rightarrow 0} \frac{d\left(X_{0}, X_{t}\right)}{h(t)}
$$

for appropriate functions $h$.

(c) 2003 Elsevier SAS. All rights reserved.

E-mail address: 1sc@math.cornell.edu (L. Saloff-Coste).

1 Research partially supported by NSF grant DMS 0102126. 


\section{Résumé}

Nous étudions la régularité intrinséque de certains mouvements browniens sur le tore de dimension infinie $\mathbb{T}^{\infty}$. Par régularité intrinséque, nous entendons régularité par rapport à la distance intrinséque associée au mouvement brownien en question. Nous montrons que certains mouvements browniens sur $\mathbb{T}^{\infty}$ satisfont la loi du logarithme itéré, c'est à dire,

$$
\limsup _{t \rightarrow 0} \frac{d\left(X_{0}, X_{t}\right)}{\sqrt{4 t \log \log (1 / t)}}=1
$$

alors que d'autres ne la satisfont pas. Par exemple, pour chaque fonction $\psi$ à variation lente telle que $\psi(t) \geqslant \log \log t$ à l'infini, nous donnons des exemples de mouvements browniens sur $\mathbb{T}^{\infty}$ tels que

$$
0<\limsup _{t \rightarrow 0} \frac{d\left(X_{0}, X_{t}\right)}{\sqrt{4 t \psi(1 / t)}}<\infty .
$$

Nous donnons des résultats similaires pour le module de continuité uniforme sur $[0,1]$. Plus précisèment, nous montrons l'existence de mouvements browniens sur $\mathbb{T}^{\infty}$ pour lesquels le résultat classique de Lévy est satisfait et, pour chaque fonction $\psi$ à variation lente telle que $\psi(t) \geqslant \log t$ à l'infini, nous donnons des exemples de mouvements browniens sur $\mathbb{T}^{\infty}$ tels que

$$
0<\lim _{\varepsilon \rightarrow 0} \sup _{\substack{0<s<t \leqslant 1 \\ t-s \leqslant \varepsilon}} \frac{d\left(X_{s}, X_{t}\right)}{\sqrt{4 t \psi(1 / t)}}<\infty .
$$

Nous donnons aussi quelques résultats partiels concernant

$$
\liminf _{t \rightarrow 0} \frac{d\left(X_{0}, X_{t}\right)}{h(t)}
$$

lorsque $h$ est une fonction appropriée.

(c) 2003 Elsevier SAS. All rights reserved.

MSC: primary 60G17, 60B15, 60J60

Keywords: Sample paths; Modulus of continuity

\section{Introduction}

Let $M$ be a compact topological space equipped with a Borel measure $\mu$ having full support. Consider a regular strictly local Dirichlet space (see [15] for background) and the associated diffusion process $\left(\Omega, X, \mathbb{P}_{x}\right)$ (if $-L$ denotes the infinitesimal generator, we make the convention that $X$ is driven by $L$, not $(1 / 2) L)$. Let $d(x, y)$ denote the corresponding intrinsic distance on $M$ and assume that $d$ defines the topology of $M$ (see, e.g., [11,13,20] for background). Under such circumstances, it is natural to study the regularity properties of the sample paths $t \mapsto X_{t}(\omega)$ in terms of the intrinsic distance $d$.

For instance, let $M=\mathbb{T}=(-\pi, \pi]$ be the circle and $\mu$ the normalized Haar (i.e., Lebesgue) measure. Consider the Dirichlet form

$$
\mathcal{E}(f, f)=\int_{\mathbb{T}}\left|f^{\prime}\right|^{2} \mathrm{~d} \mu .
$$

Then the process $X$ is the Brownian motion driven by $\left(\frac{\mathrm{d}}{\mathrm{d} x}\right)^{2}$. The intrinsic distance is given by

$$
d(x, y)=\min \{|x-y|,|x-y-2 \pi|\} .
$$

The Lévy-Khintchine law of the iterated logarithm asserts that, $\mathbb{P}_{0}$ almost surely,

$$
\limsup _{t \rightarrow 0} \frac{d\left(X_{0}, X_{t}\right)}{\sqrt{4 t \log \log 1 / t}}=1
$$


whereas Lévy's result on the modulus of continuity of Brownian paths asserts that, $\mathbb{P}_{0}$ almost surely,

$$
\lim _{\varepsilon \rightarrow 0} \sup _{\substack{0<s<t \leqslant 1 \\ t-s \leqslant \varepsilon}} \frac{d\left(X_{s}, X_{t}\right)}{\sqrt{4(t-s) \log (1 /(t-s))}}=1 .
$$

See $[18,19]$.

These two results generalize to a variety of cases including Riemannian Brownian motions and sub-elliptic diffusions on compact manifolds. See, e.g., [8]. However, the authors have shown in [7] that different behaviors occur in certain infinite dimensional situations. More precisely, for any $\lambda \in(0,1)$, [7] exhibits Brownian motions on the infinite dimensional torus such that the associated intrinsic distance $d$ is continuous and defines the topology of $\mathbb{T}^{\infty}$ and for which, $\mathbb{P}_{e}$ almost surely,

$$
0<\liminf _{t \rightarrow 0} \frac{d\left(X_{0}, X_{t}\right)}{t^{(1-\lambda) / 2}} \leqslant \limsup _{t \rightarrow 0} \frac{d\left(X_{0}, X_{t}\right)}{t^{(1-\lambda) / 2}}<\infty
$$

and

$$
0<\lim _{\varepsilon \rightarrow 0} \sup _{\substack{0<s<t \leqslant 1 \\ t-s \leqslant \varepsilon}} \frac{d\left(X_{s}, X_{t}\right)}{\sqrt{(t-s)^{(1-\lambda) / 2}}}<\infty .
$$

A natural question raised by the results of [7] is whether or not there are Brownian motions on $\mathbb{T}^{\infty}$ such that $\mathbb{P}_{e}$ almost surely,

$$
\limsup _{t \rightarrow 0} \frac{d\left(X_{0}, X_{t}\right)}{\sqrt{4 t \log \log 1 / t}}=1
$$

and/or

$$
\lim _{\varepsilon \rightarrow 0} \sup _{\substack{0<s<t \leqslant 1 \\ t-s \leqslant \varepsilon}} \frac{d\left(X_{s}, X_{t}\right)}{\sqrt{4(t-s) \log (1 /(t-s))}}=1 .
$$

In view of [7], a naive conjecture is that such Brownian motions do not exist.

The aim of this paper is to study this question and prove that, in fact, such Brownian motions do exist. Thus there is a sort of phase transition among Brownian motions on $\mathbb{T}^{\infty}$ between those which satisfy the classic law of the iterated logarithm and those which do not. There is also a similar phase transition between those Brownian motions which satisfy the classic Lévy modulus of continuity and those which do not. Our results show that these two "phase transitions" are distinct.

For technical reasons, the results presented in this paper are obtained only for a rather restricted class of Brownian motions on $\mathbb{T}^{\infty}$ (see Section 2 below). Even in this restricted context many open questions remain concerning the regularity of the sample paths of these processes. Extending our results to general Brownian motions on $\mathbb{T}^{\infty}$ (see [4,6]) and to Brownian motions on compact connected groups (see [5-7]) is an open problem. In this direction, we conjecture that any connected locally connected compact metrizable group carries a Brownian motion satisfying (1.1) and (1.2).

\section{Notation}

Let $\mathbb{T}=\mathbb{R} / 2 \pi \mathbb{Z}$ be the circle group. Let $\mathbb{T}^{\infty}=\prod_{1}^{\infty} \mathbb{T}_{i}$ be the countable product of circles $\mathbb{T}_{i}$. The group $\mathbb{T}^{\infty}$ is equipped with its natural topology (the product topology) and its normalized Haar measure $\mu$. Denote by $\mathcal{C}$ the set of all smooth functions on $\mathbb{T}^{\infty}$ which depend only on a finite number of coordinates (i.e., smooth cylindrical functions). 
On the circle $\mathbb{T}$, denote by $v_{t}$ the standard heat kernel measure associated to the infinitesimal generator $\left(\frac{\mathrm{d}}{\mathrm{d} x}\right)^{2}$. The measure $v_{t}$ has a smooth positive density $x \mapsto v_{t}(x)$ with respect to the Haar measure on $\mathbb{T}=(-\pi, \pi]$. This density is given by

$$
v_{t}(x)=\left(\frac{\pi}{t}\right)^{1 / 2} \sum_{k \in 2 \pi \mathbb{Z}} \exp \left(-\frac{|x+k|^{2}}{4 t}\right) .
$$

In words, $v_{t}$ is obtained by wrapping around the circle the Gauss measure of variance $2 t$ on $\mathbb{R}$ whose density with respect to Lebesgue measure is

$$
\left(\frac{1}{4 \pi t}\right)^{1 / 2} \exp \left(-\frac{|x|^{2}}{4 t}\right)
$$

The convolution semigroup $\left(v_{t}\right)_{t>0}$ is associated with a stochastic process $\xi=\left(\xi_{t}\right)_{t \geqslant 0}$ which is simply Brownian motion runned at twice the usual speed in classic probabilist notation and wrapped around the circle.

For any fixed sequence $\mathbf{a}=\left(a_{1}, \ldots\right)$ of positive numbers, we consider the product measures

$$
\mu_{t}=\mu_{t}^{\mathbf{a}}=\bigotimes_{1}^{\infty} v_{a_{i} t}
$$

Thus $\mu_{t}$ is a product of circle Gaussian measures. Of course, the family $\left(\mu_{t}\right)_{t>0}$ forms a convolution semigroup of measures on $\mathbb{T}^{\infty}$ and $\mu_{t}$ is the marginal at time $t$ of a diffusion process $X=X^{\mathbf{a}}=\left(X_{t}\right)_{t} \geqslant 0$ which is simply the product of independent circle Brownian motions $X^{i}=\left(X_{t}^{i}\right)_{t \geqslant 0}$ where $X_{t}^{i}=\xi_{a_{i} t}$. The infinitesimal generator driving the process $X^{\mathbf{a}}$ is the second order differential operator

$$
\sum_{1}^{\infty} a_{i} \partial_{i}^{2}
$$

whose action on any smooth cylindrical function $f \in \mathcal{C}$ is easy to compute.

The present work focusses on properties of the semigroups $\left(\mu_{t}^{\mathbf{a}}\right)_{t>0}$ and the associated processes $X^{\mathbf{a}}$. For obvious reasons, we call these processes diagonal Brownian motions on $\mathbb{T}^{\infty}$. Diagonal Gaussian semigroups and processes on $\mathbb{T}^{\infty}$ where first studied in [1,9]. For details on general Brownian motions and associated Gaussian semigroups on $\mathbb{T}^{\infty}$ see, e.g., $[2,6,17]$. From the viewpoint of Dirichlet spaces, the process $X^{\mathbf{a}}$ is associated with the Dirichlet space $(\mathcal{E}, \mathcal{D})$ where $\mathcal{D} \subset L^{2}\left(\mathbb{T}^{\infty}, \mu\right)$ is the domain of the form $\mathcal{E}$ given by

$$
\forall f \in \mathcal{C}, \quad \mathcal{E}(f, f)=\int_{\mathbf{T}^{\infty}} \sum_{1}^{\infty} a_{i}\left|\partial_{i} f\right|^{2} \mathrm{~d} \mu
$$

and $\mathcal{C} \subset \mathcal{D}$ is a core for this Dirichlet form. The intrinsic distance can then be defined by setting

$$
d(x, y)=d^{\mathbf{a}}(x, y)=\sup \left\{f(x)-f(y): f \in \mathcal{C}, \sum_{1}^{\infty} a_{i}\left|\partial_{i} f\right|^{2} \leqslant 1\right\} .
$$

As $d$ is always invariant by translation, we set

$$
d(x)=d^{\mathbf{a}}(x)=d^{\mathbf{a}}(e, x),
$$

where $e$ is the neutral element in $\mathbb{T}^{\infty}$ (i.e., $e=(0,0, \ldots)$ ). In many cases, the intrinsic distance $d$ is not a distance and may take the value $+\infty$. However, it is always symmetric and satisfies the triangle inequality. As explained in the introduction, we are only interested in cases where $d$ is actually a continuous function and defines the topology of $\mathbb{T}^{\infty}$. It is not hard to see $[2,4]$ that $d$ is given by

$$
d(x)=\left(\sum_{1}^{\infty} \frac{\left|x_{i}\right|^{2}}{a_{i}}\right)^{1 / 2},
$$


where $x=\left(x_{i}\right)_{1}^{\infty} \in \mathbb{T}^{\infty}$ with $x_{i} \in(-\pi, \pi]$. Hence $d$ is continuous and defines the topology of $\mathbb{T}^{\infty}$ if and only if

$$
\sum_{1}^{\infty} \frac{1}{a_{i}}<\infty
$$

This condition is assumed to hold throughout this article. Under this condition, we can define the volume function

$$
V(r)=V^{\mathbf{a}}(r)=\mu(B(r)),
$$

where

$$
B(r)=B^{\mathbf{a}}(r)=\left\{x: d^{\mathbf{a}}(x)<r\right\}
$$

is the open ball of radius $r$.

It turns out (see [4]) that (2.3) implies that, for any $t>0$, the measure $\mu_{t}=\mu_{t}^{\mathbf{a}}$ is absolutely continuous with respect to the Haar measure $\mu$ and admits a continuous density $x \mapsto \mu_{t}(x)=\mu_{t}^{\mathbf{a}}(x)$ given by

$$
\mu_{t}(x)=\prod_{1}^{\infty} v_{a_{i} t}\left(x_{i}\right) .
$$

Given a sequence $\mathbf{a}=\left(a_{i}\right)_{1}^{\infty}$ of positive numbers, define

$$
N(s)=N^{\mathbf{a}}(s)=\#\left\{i: a_{i} \leqslant s\right\} .
$$

This function will play a crucial role in the sequel. See also [2-4,6]. In particular we note that, by integration by parts,

$$
\sum_{1}^{\infty} \frac{1}{a_{i}}=\int_{0}^{\infty} \frac{\mathrm{d} N(s)}{s}=\int_{0}^{\infty} N(s) \frac{\mathrm{d} s}{s^{2}} .
$$

Hence (2.3) is equivalent to

$$
\int_{0}^{\infty} N(s) \frac{\mathrm{d} s}{s^{2}}<\infty .
$$

\section{On-diagonal heat kernel behavior}

Let $\left(\mu_{t}^{\mathbf{a}}\right)_{t>0}$ be a semigroup as considered above. Let us define $\mu_{t}^{\mathbf{a}}(e) \in(0, \infty]$ as follows: if $\mu_{t}^{\mathbf{a}}$ is not absolutely continuous w.r.t. Haar measure then $\mu_{t}^{\mathbf{a}}(e)=\infty$. If $\mu_{t}^{\mathbf{a}}$ is absolutely continuous with density $x \mapsto \mu_{t}^{\mathbf{a}}(x)$ (defined almost everywhere), set

$$
\mu_{t}^{\mathbf{a}}(e)=\sup _{x \in \mathbb{T}^{\infty}} \mu_{t}^{\mathbf{a}}(x)
$$

where sup means essential supremum. Note that if $\mu_{t}^{\mathbf{a}}$ admits a continuous density and $x \mapsto \mu_{t}^{\mathbf{a}}(x)$ denotes this continuous density defined everywhere then (3.8) holds true. Moreover, if $\mu_{t}^{\mathbf{a}}$ is absolutely continuous w.r.t. Haar measure for all $t>0$ then it actually admits a continuous density for all $t>0$. See [2, Theorem 4.3.2].

The function $N^{\mathbf{a}}$ defined at (2.6) can be used to compute the value of $\mu_{t}^{\mathbf{a}}(e)$ in a very precise way.

Theorem 3.1 [3,4,6]. There exists a continuous positive function $G$ with the properties that

$$
G(s)= \begin{cases}\frac{1}{2}+\mathrm{o}\left(\mathrm{e}^{-1 / s}\right) & \text { as } s \rightarrow 0, \\ \mathrm{o}\left(\mathrm{e}^{-s / 2}\right) & \text { as } s \rightarrow \infty\end{cases}
$$


and such that, for any sequence of positive numbers $\mathbf{a}=\left(a_{i}\right)_{1}^{\infty}$, we have

$$
\log \mu_{t}^{\mathbf{a}}(e)=\int_{0}^{\infty} N^{\mathbf{a}}(s / t) G(s) \frac{\mathrm{d} s}{s} .
$$

In particular, $\mu_{t}^{\mathbf{a}}$ admits a continuous density for all $t>0$ if and only if $\log N^{\mathbf{a}}(s)=\mathrm{o}(s)$ as $s$ tends to infinity.

In fact the function $G$ is explicitely given by

$$
G(s)=-s \frac{\mathrm{d}}{\mathrm{d} s} \log v_{s}(0) .
$$

To illustrate this result we recall the following classical definition. A function $f:(0, \infty) \rightarrow(0, \infty)$ is slowly varying at infinity if

$$
\forall z \in(0, \infty), \quad \lim _{s \rightarrow \infty} \frac{f(z s)}{f(s)}=1 .
$$

We will often use the fact that for any slowly varying function $f$ and for any $\varepsilon>0$,

$$
f(x) \leqslant 2 \max \left\{(x / y)^{\varepsilon},(y / x)^{\varepsilon}\right\} f(y)
$$

for all $x, y$ large enough. See [10, Theorem 1.5.6]. A function $f$ is regularly varying of index $\alpha \in(-\infty, \infty)$ if $t \mapsto t^{-\alpha} f(t)$ is slowly varying. Let $\mathcal{R}_{\alpha}$ be the class of all regularly varying functions of index $\alpha$.

Given a function $f:(0, \infty) \rightarrow(0, \infty)$, define the transform $f^{\#}$ by

$$
f^{\#}(z)=\int_{0}^{z} f(x) \frac{\mathrm{d} x}{x} .
$$

Corollary 3.2 [3]. Fix $\alpha \in[0, \infty)$. Let $\mathbf{a}=\left(a_{i}\right)_{1}^{\infty}$ be a sequence of positive reals such that $N=N^{\mathbf{a}} \in \mathcal{R}_{\alpha}$. Let $x \mapsto \mu_{t}(x)$ be the continuous density of the measure $\mu_{t}=\mu_{t}^{\mathbf{a}}$. Then, as $t \rightarrow 0$, we have

$$
\log \mu_{t}(e) \sim \begin{cases}(1 / 2) N^{\#}(1 / t) & \text { if } \alpha=0, \\ c_{\alpha} N(1 / t) & \text { if } \alpha \in(0, \infty),\end{cases}
$$

where $c_{\alpha}=\int_{0}^{\infty} s^{-1+\alpha} G(s) \mathrm{d} s$ with $G$ as in (3.10).

The following proposition will be useful in the sequel.

Proposition 3.3. Let $\mathbf{a}=\left(a_{i}\right)_{1}^{\infty}$ be a sequence of positive reals such that $N=N^{\mathbf{a}} \in \mathcal{R}_{0}$. Let $x \mapsto \mu_{t}(x)$ be the continuous density of the measure $\mu_{t}=\mu_{t}^{\mathbf{a}}$. Then, for any $c_{0}>0$, there exists a continuous positive function $\eta$ satisfying $\lim _{s \rightarrow 0} \eta(s)=0$ and such that, for all $c \geqslant c_{0}$ and all $t \in\left(0,1 / a_{1}\right)$, we have

$$
\left|\log \frac{\mu_{t}(e)}{\mu_{c t}(e)}-\frac{1}{2}(\log c) N(1 / t)\right| \leqslant|1-c| N(1 / t) \eta(t) .
$$

Proof. Let $G$ be the function introduced in Theorem 3.9 and defined at (3.10). Then

$$
\begin{aligned}
\log \frac{\mu_{t}(e)}{\mu_{c t}(e)} & =\log \mu_{t}(e)-\log \mu_{c t}(e) \\
& =\int_{0}^{\infty} N(s / t)(G(s)-G(c s)) \frac{\mathrm{d} s}{s}
\end{aligned}
$$




$$
\begin{aligned}
& =N(1 / t) \int_{0}^{\infty} \frac{N(s / t)}{N(1 / t)}(G(s)-G(c s)) \frac{\mathrm{d} s}{s} \\
& \sim N(1 / t) \int_{0}^{\infty}(G(s)-G(c s)) \frac{\mathrm{d} s}{s} \quad \text { as } t \rightarrow 0 .
\end{aligned}
$$

Next, we compute

$$
\begin{aligned}
\int_{0}^{\infty}(G(s)-G(c s)) \frac{\mathrm{d} s}{s} & =\lim _{\varepsilon \rightarrow 0} \int_{\varepsilon}^{\infty}(G(s)-G(c s)) \frac{\mathrm{d} s}{s}=\lim _{\varepsilon \rightarrow 0} \int_{\varepsilon}^{c \varepsilon} G(s) \frac{\mathrm{d} s}{s} \\
& =\lim _{\varepsilon \rightarrow 0}\left(\frac{1}{2} \int_{\varepsilon}^{c \varepsilon} \frac{\mathrm{d} s}{s}+\int_{\varepsilon}^{c \varepsilon}\left(G(s)-\frac{1}{2}\right) \frac{\mathrm{d} s}{s}\right) \\
& =\frac{1}{2} \log c .
\end{aligned}
$$

Hence, for all $t \in\left(0,1 / a_{1}\right)$,

$$
\begin{aligned}
\log \frac{\mu_{t}(e)}{\mu_{c t}(e)}-\frac{1}{2}(\log c) N(1 / t) & =N(1 / t) \int_{0}^{\infty}\left(\frac{N(s / t)}{N(1 / t)}-1\right)(G(s)-G(c s)) \frac{\mathrm{d} s}{s} \\
& =(1-c) N(1 / t) \int_{0}^{\infty}\left(\frac{N(s / t)}{N(1 / t)}-1\right)\left(\frac{G(s)-G(c s)}{(1-c) s}\right) \mathrm{d} s .
\end{aligned}
$$

Now we want to bound

$$
I(t, c)=\int_{0}^{\infty}\left|\frac{N(s / t)}{N(1 / t)}-1\right|\left|\frac{G(s)-G(c s)}{(1-c) s}\right| \mathrm{d} s .
$$

Set

$$
U_{c}(t)=\sup \left\{\left|G^{\prime}(s)\right|: s \text { between } t \text { and } c t\right\} .
$$

Then we have

$$
I(t, c) \leqslant \int_{0}^{\infty}\left|\frac{N(s / t)}{N(1 / t)}-1\right| U_{c}(s) \mathrm{d} s .
$$

We need the following lemma.

Lemma 3.4. The function $G$ satisfies:

(1) $G^{\prime}(s)=-2 s \mathrm{e}^{-s}(1+\mathrm{o}(1))$ as $s \rightarrow \infty$.

(2) $G^{\prime}(s)=-(2 / s) \mathrm{e}^{-\pi^{2} / s}(1+\mathrm{o}(1))$ as $s \rightarrow 0$.

Proof. The first statement follows from (3.10) and the Poisson formula

$$
v_{t}(0)=\sum_{k \in \mathbb{Z}} \mathrm{e}^{-k^{2} t}
$$


The second statement follows from the first one and the relation (see [4, Lemma 5.18])

$$
G(s)+G\left(\pi^{2} / s\right)=1 / 2 .
$$

To finish the proof of Proposition 3.3, set $M_{0}=\sup \left|G^{\prime}\right|$ and let $s_{0}$ be the smallest real such that $\left|G^{\prime}(s)\right| \leqslant \mathrm{e}^{-s / 2}$ for $s \geqslant s_{0}$. Lemma 3.4 implies that $M_{0}, s_{0}$ are finite. Moreover, for any $c_{0} \in(0,1)$,

$$
\forall c \in\left(c_{0}, \infty\right), \forall t>0, \quad U_{c}(t) \leqslant M_{0} \mathrm{e}^{\left(s_{0}-c_{0} t\right) / 2} .
$$

Hence

$$
I(t, c) \leqslant \eta(t)=M_{0} \mathrm{e}^{s_{0}} \int_{0}^{\infty}\left|\frac{N(s / t)}{N(1 / t)}-1\right| \mathrm{e}^{-c_{0} s / 2} \mathrm{~d} s .
$$

Now, as $N$ is slowly varying, we have

$$
\lim _{t \rightarrow 0} \frac{N(s / t)}{N(1 / t)}=1
$$

and, for any $\varepsilon>0$, there exists a constant $C_{\varepsilon}$ such that (see (3.11))

$$
\forall t \in\left(0,1 / a_{1}\right), \forall s>0, \quad\left|\frac{N(s / t)}{N(1 / t)}-1\right| \leqslant C_{\varepsilon}\left(1+s^{\varepsilon}\right) .
$$

Thus the dominated convergence theorem shows that $\lim _{t \rightarrow 0} \eta(t)=0$ as desired.

\section{Mean square displacement and statement of the main results}

\subsection{Mean square displacement}

One of the simplest measure of the regularity of the paths of a stochastic process is the mean square displacement $\mathbb{E}_{e}\left(d\left(X_{t}\right)^{2}\right)$. Under the normalization used here, the square mean displacement of Brownian motion on the circle is asymptotic to $2 t$ as $t$ tends to zero. More precisely, let $\xi=\left(\xi_{t}\right)_{t \geqslant 0}$ be Brownian motion on the circle, runned at twice the usual speed (i.e., driven by $\left.\left(\frac{\mathrm{d}}{\mathrm{d} x}\right)^{2}\right)$. Set

$$
\phi(t)=\mathbb{E}_{0}\left(\left|\xi_{t}\right|^{2}\right)=\frac{1}{2 \pi} \int_{-\pi}^{\pi}|x|^{2} v_{t}(x) \mathrm{d} x
$$

where $|x|$ denote the distance from the neutral element on the circle. Then $\phi(s) \sim 2 s$ as $s$ tends to zero and $\phi(s) \sim \pi^{2} / 3$ as $s$ tends to $\infty$. Define the function $\Phi$ by

$$
\Phi(t)=-\frac{\mathrm{d}}{\mathrm{d} t}\left(\frac{\phi(t)}{t}\right)
$$

For diagonal Brownian motion on $\mathbb{T}^{\infty}$, asymptotically as $t$ tends to 0 , the mean square displacement is always greater than $2 t$.

Theorem 4.1. Let $\mathbf{a}=\left(a_{i}\right)_{1}^{\infty}$ be a sequence of positive numbers such that $N=N^{\mathbf{a}}$ is regularly varying of index $\lambda \in[0,1)$. Let $X=X^{\mathbf{a}}=\left(X_{t}\right)_{t>0}$ be the associated process on $\mathbb{T}^{\infty}$ (see the introduction). Then the mean square displacement satisfies

$$
\mathbb{E}_{e}\left(d\left(X_{t}\right)^{2}\right) \sim c_{\lambda} t N(1 / t) \quad \text { as } t \rightarrow 0,
$$


where $c_{\lambda}=\int_{0}^{\infty} s^{\lambda} \Phi(s) \mathrm{d}$ s. In particular, if $N$ is slowly varying (i.e., $\left.\lambda=0\right)$ then

$$
\mathbb{E}_{e}\left(d\left(X_{t}\right)^{2}\right) \sim 2 t N(1 / t) \quad \text { as } t \rightarrow 0 .
$$

Remark. For $\lambda \in(0,1)$, we have

$$
c_{\lambda}=\int_{0}^{\infty} s^{\lambda} \Phi(s) \mathrm{d} s=\frac{4 \lambda \Gamma(\lambda)}{1-\lambda} \sum_{1}^{\infty} \frac{(-1)^{n+1}}{n^{2 \lambda}} \sim \frac{\pi^{2}}{3(1-\lambda)} \quad \text { as } \lambda \rightarrow 1 .
$$

In particular, this shows that $c_{\lambda} \rightarrow \infty$ as $\lambda \rightarrow 1$. This formula follows from a rather nontrivial computation given in the appendix.

Proof of Theorem 4.1. We write

$$
\begin{aligned}
\mathbb{E}_{e}\left(d\left(X_{t}\right)^{2}\right) & =\sum_{i=1}^{\infty} \frac{1}{a_{i}} \mathbb{E}_{0}\left(\left|\xi_{a_{i}}\right|^{2}\right)=\sum_{1}^{\infty} \frac{1}{a_{i}} \phi\left(a_{i} t\right) \\
& =\int_{0}^{\infty} \phi(s t) \frac{\mathrm{d} N(s)}{s}=t \int_{0}^{\infty} \frac{\phi(s)}{s} \mathrm{~d} N(s / t) \\
& =t \int_{0}^{\infty} N(s / t) \Phi(s) \mathrm{d} s=t N(1 / t)\left(\int_{0}^{\infty} \frac{N(s / t)}{N(1 / t)} \Phi(s) \mathrm{d} s\right) \\
& \sim t N(1 / t)\left(\int_{0}^{\infty} s^{\lambda} \Phi(s) \mathrm{d} s\right) \quad \text { as } t \rightarrow 0 .
\end{aligned}
$$

The last step uses the fact that $N$ is regularly varying of index $\lambda$ and we have used the fact that the continuous function $\Phi$ is integrable, see below. The next lemma gathers the necessary properties of the functions $\phi$ and $\Phi$.

\section{Lemma 4.2. We have}

$$
\lim _{s \rightarrow \infty} \phi(s)=\pi^{2} / 3, \quad \lim _{s \rightarrow \infty} s^{2} \Phi(s)=\pi^{2} / 3
$$

and

$$
\frac{\phi(s)}{2 s}=1+\mathrm{o}\left(\mathrm{e}^{-1 / s}\right), \quad \Phi(s)=\mathrm{o}\left(s^{-1} \mathrm{e}^{-1 / s}\right) \quad \text { as } s \rightarrow 0 .
$$

Proof. The first assertion concerning $\phi$ easily follows from the fact that $v_{s}(x) \rightarrow 1$ as $s$ tends to infinity. Then we have

$$
\Phi(s)=\frac{\phi(s)}{s^{2}}-\frac{\phi^{\prime}(s)}{s} .
$$

By integration by parts and the fact that $v_{t}$ solves the heat equation on $\mathbb{T}$, we have

$$
\phi^{\prime}(s)=\frac{1}{2 \pi} \int_{-\pi}^{\pi} x^{2} \frac{\mathrm{d}}{\mathrm{d} s} v_{s}(x) \mathrm{d} x=\frac{1}{2 \pi} \int_{-\pi}^{\pi} x^{2} \frac{\mathrm{d}^{2}}{\mathrm{~d} x^{2}} v_{s}(x) \mathrm{d} x=2-2 v_{s}(\pi) .
$$

Hence the second assertion about $\phi$ gives

$$
\Phi(s)=2 s^{-1} v_{s}(\pi)-s^{-1}\left(2-s^{-1} \phi(s)\right)=\mathrm{o}\left(s^{-1} \mathrm{e}^{-1 / s}\right) \quad \text { as } s \rightarrow 0 .
$$


To prove the second assertion about $\phi$, we write

$$
\begin{aligned}
1-\frac{\phi(s)}{2 s} & =1-\frac{1}{2 \pi s} \int_{0}^{\pi} x^{2} v_{s}(x) \mathrm{d} x \\
& =1-\frac{1}{2 \pi s} \int_{0}^{\pi} x^{2} \sqrt{\frac{\pi}{s}} \mathrm{e}^{-|x|^{2} / 4 s} \mathrm{~d} x+\mathrm{o}\left(\mathrm{e}^{-1 / s}\right) \\
& =1-\frac{1}{\sqrt{4 \pi}} \int_{0}^{\pi / \sqrt{s}} x^{2} \mathrm{e}^{-|x|^{2} / 4} \mathrm{~d} x+\mathrm{o}\left(\mathrm{e}^{-1 / s}\right) \\
& =\frac{1}{\sqrt{4 \pi}} \int_{\pi / \sqrt{s}}^{\infty} x^{2} \mathrm{e}^{-|x|^{2} / 4} \mathrm{~d} x+\mathrm{o}\left(\mathrm{e}^{-1 / s}\right)=\mathrm{o}\left(\mathrm{e}^{-1 / s}\right) .
\end{aligned}
$$

Finally, we have

$$
\frac{\left|\phi^{\prime}(s)\right|}{s}=\frac{2}{s}\left|1-v_{s}(\pi)\right|=\mathrm{o}\left(\mathrm{e}^{-s / 2}\right)
$$

as $s$ tends to infinity. This gives the last desired result concerning $\Phi$ and finishes the proof of Lemma 4.2.

\subsection{Main results}

This section gathers our main results in one theorem, Theorem 4.4 below. More precise versions are stated and proved later in the paper. The notation is as follows. We consider sequences $\mathbf{a}=\left(a_{i}\right)_{1}^{\infty}$ of positive numbers whose growth is such that the measure $\mu_{t}=\mu_{t}^{\mathbf{a}}$ admits a continuous density $x \mapsto \mu_{t}(x)$ and the intrinsic distance $d=d^{\mathbf{a}}$ defines the topology of $\mathbb{T}^{\infty}$. By [2,4], this is the case when $N=N^{\mathbf{a}} \in \mathcal{R}_{\lambda}$ with $\lambda \in[0,1)$. For comparison, we first recall a result from [7].

Theorem 4.3 [7]. Let $\mathbf{a}=\left(a_{i}\right)_{1}^{\infty}$ be a sequence of positive numbers such that $N=N^{\mathbf{a}}$ is regularly varying of index $\lambda \in(0,1)$. Then $\log \mu_{t}(e) \sim c_{\lambda} N(1 / t)$ as $t$ tends to 0 and the sample paths of the process $X=X^{\mathbf{a}}$ have the following properties:

(1) $\mathbb{P}_{e}$ almost surely,

$$
0<\liminf _{t \rightarrow 0} \frac{d\left(X_{t}\right)}{\sqrt{t N(1 / t)}} \leqslant \limsup _{t \rightarrow 0} \frac{d\left(X_{t}\right)}{\sqrt{t N(1 / t)}}<\infty .
$$

(2) $\mathbb{P}_{e}$ almost surely,

$$
0<\lim _{\varepsilon \rightarrow 0} \sup _{\substack{0<s<t \leqslant 1 \\ t-s \leqslant \varepsilon}} \frac{d\left(X_{s}, X_{t}\right)}{\sqrt{(t-s) N(1 /(t-s))}}<\infty .
$$


Observe that in this case, the behavior of the sample paths can be read off from the behavior of $\log \mu_{t}(e)$ in a very simple way. In the following theorem this is not the case.

Theorem 4.4. Let $\mathbf{a}=\left(a_{i}\right)_{1}^{\infty}$ be a sequence of positive numbers such that $N=N^{\mathbf{a}}$ is slowly varying. Then $\log \mu_{t}(e) \sim(1 / 2) N^{\#}(1 / t)$ (see (3.12)) as t tends to 0 and the sample paths of the process $X^{\mathbf{a}}$ have the following properties.

(1) We always have, $\mathbb{P}_{e}$ almost surely,

$$
\liminf _{t \rightarrow 0} \frac{d\left(X_{t}\right)}{\sqrt{t N(1 / t)}}<\infty
$$

(2) If $N(s)=\mathrm{o}(\log \log s)$ at infinity then, $\mathbb{P}_{e}$ almost surely,

$$
\begin{aligned}
& \limsup _{t \rightarrow 0} \frac{d\left(X_{t}\right)}{\sqrt{4 t \log \log 1 / t}}=1, \\
& \lim _{\varepsilon \rightarrow 0} \sup _{\substack{0<s<t \leqslant 1 \\
t-s \leqslant \varepsilon}} \frac{d\left(X_{s}, X_{t}\right)}{\sqrt{4(t-s) \log (1 /(t-s))}}=1
\end{aligned}
$$

and

$$
\liminf _{t \rightarrow 0} \frac{d\left(X_{t}\right)}{\sqrt{4 t \log \log 1 / t}}=0 .
$$

(3) If $N(s)=\mathrm{o}(\log s)$ and $\log \log s=\mathrm{O}(N(s))$ at infinity then, $\mathbb{P}_{e}$ almost surely,

$$
0<\liminf _{t \rightarrow 0} \frac{d\left(X_{t}\right)}{\sqrt{t N(1 / t)}} \leqslant \limsup _{t \rightarrow 0} \frac{d\left(X_{t}\right)}{\sqrt{t N(1 / t)}}<\infty,
$$

and

$$
\lim _{\varepsilon \rightarrow 0} \sup _{\substack{0<s<t \leqslant 1 \\ t-s \leqslant \varepsilon}} \frac{d\left(X_{s}, X_{t}\right)}{\sqrt{4(t-s) \log (1 /(t-s))}}=1 .
$$

(4) If $\log \log s=\mathrm{O}(N(s))$ at infinity then, $\mathbb{P}_{e}$ almost surely,

$$
0<\liminf _{t \rightarrow 0} \frac{d\left(X_{t}\right)}{\sqrt{t N(1 / t)}} \leqslant \limsup _{t \rightarrow 0} \frac{d\left(X_{t}\right)}{\sqrt{t N(1 / t)}}<\infty .
$$

(5) If $\log s=\mathrm{O}(N(s))$ at infinity then, $\mathbb{P}_{e}$ almost surely,

$$
0<\lim _{\varepsilon \rightarrow 0} \sup _{\substack{0<s<t \leqslant 1 \\ t-s \leqslant \varepsilon}} \frac{d\left(X_{s}, X_{t}\right)}{\sqrt{4(t-s) N(1 /(t-s))}}<\infty .
$$

Note that the different cases in this theorem are not exclusive. Roughly speaking, for a slowly varying $N$, there are three cases to consider:

(a) If $N$ is smaller than $\log \log$, then we obtain a classical Lévy-Khintchine law of iterated logarithm

$$
\limsup _{t \rightarrow 0} \frac{d\left(X_{t}\right)}{\sqrt{4 t \log \log 1 / t}}=1
$$

and a classical Lévy modulus of continuity

$$
\lim _{\varepsilon \rightarrow 0} \sup _{\substack{0<s<t \leqslant 1 \\ t-s \leqslant \varepsilon}} \frac{d\left(X_{s}, X_{t}\right)}{\sqrt{4(t-s) \log (1 /(t-s))}}=1 .
$$


(b) If $N$ is larger than $\log \log$ but smaller than $\log$, then we still have a classical Lévy modulus of continuity, but the Lévy-Khintchine-type result is not classical any more: $d\left(X_{t}\right)$ is now controled by the function $\sqrt{t N(1 / t)}$.

(c) If $N$ is larger than log (but still slowly varying), then all regularity behaviors are controled by the function $\sqrt{t N(1 / t)}$.

An interesting aspect of the above results concerns the behavior of

$$
\liminf _{t \rightarrow 0} \frac{d\left(X_{t}\right)}{h(t)} \text {. }
$$

Namely, when $N$ grows faster than $\log \log$, the correct function $h$ is $\sqrt{t N(1 / t)}$, a result that is quite different from what happens in finite dimension. When $N(s)=\mathrm{o}(\log \log (s))$ at infinity, finding the correct function (or class of functions) $h$ is an open problem which is quite interesting.

\section{Preliminary estimates}

\subsection{Gausssian bounds}

One of the main tools used in this paper is a precise two-sided Gaussian estimate for the density $x \mapsto \mu_{t}(x)$ of any diagonal symmetric Gaussian semigroup $\left(\mu_{t}\right)_{t>0}$ on $\mathbb{T}^{\infty}$ under the assumption that (2.3) is satisfied (compare to [3, Theorem 2.9]).

Theorem 5.1. Let $\mathbf{a}=\left(a_{i}\right)_{1}^{\infty}$ be a sequence of positive numbers satisfying (2.3). Let $x \mapsto \mu_{t}(x)$ be the density of the measure $\mu_{t}=\mu_{t}^{\mathbf{a}}$. Let $d$ be defined by (2.2) and $N$ by (2.6). Then, for all $\delta \geqslant 0, x \in \mathbb{T}^{\infty}$ and $t>0$, we have

$$
\mu_{t}(e) \exp \left(-\frac{d(x)^{2}}{4 t}\right) \leqslant \mu_{t}(x) \leqslant \mu_{t}(e) \exp \left(-\frac{d(x)^{2}}{4(1+\delta) t}+R_{t}+S_{t}^{\delta}\right),
$$

where

$$
R_{t}=\frac{3}{t} \int_{1 / t}^{\infty} \frac{\mathrm{d} N(s)}{s}, \quad S_{t}^{\delta}=\int_{0}^{1 / t} \log \left(1+\mathrm{e}^{-\delta /((1+\delta) s t)}\right) \mathrm{d} N(s) .
$$

In particular, for all $x \in \mathbb{T}^{\infty}$ and $t>0$, we have

$$
\mu_{t}(e) \exp \left(-\frac{d(x)^{2}}{4 t}\right) \leqslant \mu_{t}(x) \leqslant \mu_{t}(e) \exp \left(-\frac{d(x)^{2}}{4 t}+R_{t}+(\log 2) N(1 / t)\right) .
$$

Before giving the proof of this theorem, we state a lemma which controls $R_{t}$ and $S_{t}^{\delta}$.

Lemma 5.2. Let $R_{t}$ and $S_{t}^{\delta}$ be as in Theorem 5.1. For $\delta=0$, we have $S_{t}^{0}=(\log 2) N(1 / t)$.

(1) Assume that $N$ is a slowly varying function. Then $R_{t}=\mathrm{o}(N(1 / t))$ as $t$ tends to 0 and, for any $\delta>0$, $S_{t}^{\delta}=\mathrm{o}(N(1 / t))$ as $t$ tends to 0 .

(2) Assume that $N$ is a regularly varying function of index $\alpha \in(0,1)$. Then $R_{t} \sim r_{\alpha} N(1 / t)$ and $S_{t}^{\delta} \sim s_{\alpha}^{\delta} N(1 / t)$ as $t$ tends to 0 with

$$
r_{\alpha}=\frac{\alpha}{1-\alpha}, \quad s_{\alpha}^{\delta}=\alpha \int_{1}^{\infty} s^{\alpha-1} \log \left(1+\mathrm{e}^{-b_{\delta} s}\right) \mathrm{d} s
$$

and $b_{\delta}=\delta /(1+\delta), \delta>0$. 
Proof. For $\delta=0$, we have indeed $S_{t}^{0}=(\log 2) N(1 / t)$. Integration by parts yields

$$
\begin{aligned}
& R_{t}=N(1 / t) \int_{1}^{\infty}\left(\frac{N(s / t)}{N(1 / t)}-1\right) \frac{\mathrm{d} s}{s^{2}}, \\
& S_{t}^{\delta}=N(1 / t) \int_{0}^{1}\left(1-\frac{N(s / t)}{N(1 / t)}\right) \frac{b_{\delta} \mathrm{e}^{-b_{\delta} / s} \mathrm{~d} s}{\left(1+\mathrm{e}^{-b_{\delta} / s}\right) s^{2}} .
\end{aligned}
$$

By (3.11), if $N$ is slowly varying, we have $N(y) \leqslant C_{\gamma}\left(1+(y / x)^{\gamma}\right) N(x)$ for all $\gamma>0$ and all $x, y$ large enough. If $N$ is regularly varying of index $\alpha$ we have instead $N(y) \leqslant C_{\gamma}\left(1+(y / x)^{\gamma}\right)(y / x)^{\alpha} N(x)$ and $N(s / t) / N(1 / t) \rightarrow s^{\alpha}$ as $t \rightarrow 0$. Hence the dominated convergence theorem gives the stated results.

For the proof of Theorem 5.1 we will use the following lemma (compare to [3, Theorem 2.6]).

Lemma 5.3. On the circle $\mathbb{T}$, the heat kernel $v_{t}(x)$ at (2.1) satisfies

$$
\forall x \in[-\pi, \pi], \forall t>0, \quad v_{t}(0) \mathrm{e}^{-x^{2} / 4 t} \leqslant v_{t}(x) \leqslant v_{t}(0) \mathrm{e}^{-x^{2} /[4(1+\delta) t]}\left(1+\mathrm{e}^{-\delta /[(1+\delta) t]}\right) .
$$

Proof. Assume $x>0$ and rewrite (2.1) as

$$
v_{t}(x)=\left(\frac{\pi}{t}\right)^{1 / 2} \mathrm{e}^{-x^{2} / 4 t}\left(1+\sum_{k=1}^{\infty} \mathrm{e}^{-\pi^{2} k^{2} / t}\left(\mathrm{e}^{-\pi k x / t}+\mathrm{e}^{\pi k x / t}\right)\right) .
$$

The lower bound immediately follows. For the upper bound, write

$$
\begin{aligned}
& \sum_{k=1}^{\infty} \mathrm{e}^{-\pi^{2} k^{2} / t}\left(\mathrm{e}^{-\pi k x / t}+e^{\pi k x / t}\right) \\
& \quad=\mathrm{e}^{-\pi^{2} / t}\left(\mathrm{e}^{-\pi x / t}+\mathrm{e}^{\pi x / t}\right)+\sum_{k=2}^{\infty} \mathrm{e}^{-\pi^{2} k^{2} / t}\left(\mathrm{e}^{-\pi k x / t}+\mathrm{e}^{\pi k x / t}\right) \\
& \quad \leqslant \mathrm{e}^{-\pi^{2} / t}\left(\mathrm{e}^{-\pi x / t}+\mathrm{e}^{\pi x / t}\right)+\sum_{k=2}^{\infty} \mathrm{e}^{-\pi^{2} k^{2} / t}+\sum_{k=2}^{\infty} \mathrm{e}^{-\pi^{2} k(k-1) / t} \\
& \leqslant \mathrm{e}^{-\left(\pi^{2} / t\right)+(\pi x / t)}+\sum_{k=1}^{\infty} \mathrm{e}^{-\pi^{2} k^{2} / t}+\sum_{k=1}^{\infty} \mathrm{e}^{-\pi^{2} k(k+1) / t} \\
& \leqslant \mathrm{e}^{-\left(\pi^{2} / t\right)+(\pi x / t)}+2 \sum_{k=1}^{\infty} \mathrm{e}^{-\pi^{2} k^{2} / t} .
\end{aligned}
$$

Using this in (5.2) and the fact that

$$
v_{t}(0)=\left(\frac{\pi}{t}\right)^{1 / 2}\left(1+2 \sum_{k=1}^{\infty} \mathrm{e}^{-\pi^{2} k^{2} / t}\right)>\left(\frac{\pi}{t}\right)^{1 / 2},
$$

we obtain

$$
v_{t}(x) \leqslant\left(\frac{\pi}{t}\right)^{1 / 2} \mathrm{e}^{-x^{2} / 4 t}\left(1+2 \sum_{k=1}^{\infty} \mathrm{e}^{-\pi^{2} k^{2} / t}+\mathrm{e}^{-\left(\pi^{2} / t\right)+(\pi x / t)}\right)
$$




$$
\begin{aligned}
& \leqslant v_{t}(0) \mathrm{e}^{-x^{2} / 4 t}+v_{t}(0) \mathrm{e}^{-\left(\pi^{2} / t\right)+(\pi x / t)-\left(x^{2} / 4 t\right)} \\
& =v_{t}(0) \mathrm{e}^{-x^{2} / 4 t}+v_{t}(0) \mathrm{e}^{-x^{2} /[4(1+\delta) t]} \mathrm{e}^{-\left[\pi^{2}-\pi x+\delta x^{2} / 4(1+\delta)\right] / t} .
\end{aligned}
$$

For $0 \leqslant x \leqslant \pi, y(x)=\pi^{2}-\pi x+\delta x^{2} / 4(1+\delta)$ is decreasing. Hence $y(x) \geqslant \delta \pi^{2} / 4(1+\delta)$ and we have

$$
\begin{aligned}
v_{t}(x) & \leqslant v_{t}(0) \mathrm{e}^{-x^{2} / 4 t}+v_{t}(0) \mathrm{e}^{-x^{2} /[4(1+\delta) t]} \mathrm{e}^{-\delta \pi^{2} /[4(1+\delta) t]} \\
& \leqslant v_{t}(0) \mathrm{e}^{-x^{2} /[4(1+\delta) t]}\left(1+\mathrm{e}^{-\delta /[(1+\delta) t]}\right)
\end{aligned}
$$

This ends the proof of Lemma 5.3.

Proof of Theorem 5.1. The lower bound follows directly from the lower bound in Lemma 5.3. For the upper bound, use (2.5) and write, for $x=\left(x_{i}\right)_{1}^{\infty}, x_{i} \in[-\pi, \pi]$,

$$
\mu_{t}(x)=\prod_{1}^{\infty} v_{a_{i} t}\left(x_{i}\right)=\left(\prod_{i: a_{i} t \leqslant 1} v_{a_{i} t}\left(x_{i}\right)\right)\left(\prod_{i: a_{i} t>1} v_{a_{i} t}\left(x_{i}\right)\right) .
$$

In the first product we use Lemma 5.3 whereas, in the second product, we use $v_{a_{i}}(x) \leqslant v_{a_{i}}(0)$. This gives

$$
\prod_{i: a_{i} t \leqslant 1} v_{a_{i} t}\left(x_{i}\right) \leqslant\left(\prod_{i: a_{i} t \leqslant 1} v_{a_{i} t}(0)\right)\left(\prod_{i: a_{i} t \leqslant 1}\left(1+\mathrm{e}^{-\delta /(1+\delta) a_{i} t}\right)\right) \exp \left(-\sum_{i: a_{i} t \leqslant 1} \frac{x_{i}^{2}}{4 a_{i}(1+\delta) t}\right) .
$$

Next, observe that

$$
\sum_{i: a_{i} t \leqslant 1} \frac{x_{i}^{2}}{4 a_{i}(1+\delta) t}=\frac{d(x)^{2}}{4(1+\delta) t}-\sum_{i: a_{i} t>1} \frac{x_{i}^{2}}{4 a_{i}(1+\delta) t} \geqslant \frac{d(x)^{2}}{4(1+\delta) t}-\frac{3}{t} \sum_{i: a_{i} t>1} \frac{1}{a_{i}} .
$$

Hence

$$
\begin{aligned}
\mu_{t}(x) & \leqslant \mu_{t}(e) \exp \left(-\frac{d(x)^{2}}{4(1+\delta) t}+\frac{3}{t} \sum_{i: a_{i} t>1} \frac{1}{a_{i}}+\sum_{i: a_{i} t \leqslant 1} \log \left(1+\mathrm{e}^{-\delta /(1+\delta) a_{i} t}\right)\right) \\
& =\mu_{t}(e) \exp \left(-\frac{d(x)^{2}}{4(1+\delta) t}+R_{t}+S_{t}^{\delta}\right),
\end{aligned}
$$

as desired.

\subsection{A simple inequality}

The Gaussian lower bound of Theorem 5.1 leads to a simple but crucial inequality relating the volume function $V(r)$ to $\mu_{t}(e)$.

Proposition 5.4. Assuming that $\mu_{t}(x)$ admits a continuous density $x \mapsto \mu_{t}(x)$ w.r.t. Haar measure, we have

$$
\forall u, \tau>0, \quad \mu_{\tau}(e) V(\sqrt{u \tau}) \leqslant \mathrm{e}^{u / 4} .
$$

Proof. Integrating the lower Gaussian bound

$$
\mu_{\tau}(x) \geqslant \mu_{\tau}(e) \mathrm{e}^{-d(x)^{2} /(4 \tau)}
$$

of Theorem 5.1, we obtain

$$
1 \geqslant \mu_{\tau}(e) \int_{d(x)^{2} \leqslant u \tau} \mathrm{e}^{-d(x)^{2} / 4 \tau} \mathrm{d} \mu(x) \geqslant \mu_{\tau}(e) V(\sqrt{u \tau}) \mathrm{e}^{-u / 4} .
$$

This proves the desired inequality. 
Remark. By [4, Proposition 5.9], Proposition 5.4 holds in fact for all symmetric Gaussian semigroups on $\mathbb{T}^{\infty}$, not only for diagonal ones.

\section{Regularity of sample paths: upper bounds}

This section is devoted to upper bounds concerning the regularity of the sample paths of diagonal Brownian motions on $\mathbb{T}^{\infty}$. The techniques we use are rather classical. The primarily tool is a deviation inequality proved below and based on the Gaussian upper bound of Theorem 5.1.

\subsection{A deviation inequality}

The aim of this section is to prove the following lemma.

Lemma 6.1. Let $\mathbf{a}=\left(a_{i}\right)_{1}^{\infty}, a_{i}>0$ be such that $N=N^{\mathbf{a}}$ is slowly varying.

(1) For any $\delta>0$ there exist a constant $C_{0}=C_{0}(\delta)>0$, and $t_{0}=t_{0}(\delta) \in(0,1)$ such that, for all $t \in\left(0, t_{0}\right)$ and all $r \geqslant \sqrt{C_{0} t N(1 / t)}$, we have

$$
\mathbb{P}_{e}\left(d\left(X_{t}\right)>r\right) \leqslant\left(1+\frac{\delta}{2}\right) \exp \left(-\frac{r^{2}}{4(1+\delta) t}\right) \text {. }
$$

Moreover, $C_{0}(1 / 2)<50$ and, for all $\delta \in(0,1]$, we have $C_{0}(\delta) \leqslant 36 \delta^{-2}$.

(2) For any $\delta>0$ there exist a constant $C_{1}=C_{1}(\delta)>0$, and $t_{1}=t_{1}(\delta) \in(0,1)$ such that, for all $t \in\left(0, t_{1}\right)$ and all $r \geqslant \sqrt{C_{1} t N(1 / t)}$, we have

$$
\mathbb{P}_{e}\left(\sup _{s \in(0, t)} d\left(X_{s}\right)>r\right) \leqslant\left(2+\frac{\delta}{2}\right) \exp \left(-\frac{r^{2}}{4(1+\delta) t}\right) .
$$

Moreover, for $\delta \in(0,1]$, we have $C_{1}(\delta) \leqslant 2^{12} \delta^{-4}$.

Proof. The Gaussian upper bound of Theorem 5.1 gives

$$
\begin{aligned}
\mathbb{P}_{e}\left(d\left(X_{t}\right)>r\right) & \leqslant \mu_{t}(e) \mathrm{e}^{R_{t}+(\log 2) N(1 / t)} \int_{\{d(x)>r\}} \mathrm{e}^{-d(x)^{2} / 4 t} \mathrm{~d} \mu(x) \\
& =\mu_{t}(e) \mathrm{e}^{R_{t}+(\log 2) N(1 / t)} \int_{r^{2}}^{\infty} \mathrm{e}^{-s / 4 t} \mathrm{~d} V(\sqrt{s}) \\
& \leqslant \frac{1}{4} \mu_{t}(e) \mathrm{e}^{R_{t}+(\log 2) N(1 / t)} \int_{r^{2} / t}^{\infty} \mathrm{e}^{-s / 4} V(\sqrt{s t}) \mathrm{d} s .
\end{aligned}
$$

Choose $a=1+\delta^{-1}$ with $\delta>0$ and apply Proposition 5.4 with $u=s / a, \tau=a t$, to obtain

$$
\mathbb{P}_{e}\left(d\left(X_{t}\right)>r\right) \leqslant \frac{\mu_{t}(e) \mathrm{e}^{R_{t}+(\log 2) N(1 / t)}}{4 \mu_{a t}(e)} \int_{r^{2} / t}^{\infty} \mathrm{e}^{-(s / 4)+(s / 4 a)} \mathrm{d} s
$$




$$
\begin{aligned}
& =\frac{\mu_{t}(e) \mathrm{e}^{R_{t}+(\log 2) N(1 / t)}}{4 \mu_{a t}(e)} \int_{r^{2} / t}^{\infty} \mathrm{e}^{-s / 4(1+\delta)} \mathrm{d} s \\
& =\frac{(1+\delta) \mu_{t}(e)}{\mu_{a t}(e)} \mathrm{e}^{-r^{2} / 4(1+\delta) t+R_{t}+(\log 2) N(1 / t)} \\
& =(1+\delta) \exp \left[\log \frac{\mu_{t}(e)}{\mu_{a t}(e)}+R_{t}+(\log 2) N(1 / t)-\frac{r^{2}}{4(1+\delta) t}\right] .
\end{aligned}
$$

As $a>1$, by Lemma 5.2 and Proposition 3.3, for any given $\delta>0$, we can find $t_{0}=t_{0}(\delta)$ such that, for all $t \in\left(0, t_{0}\right)$,

$$
0<\log \frac{\mu_{t}(e)}{\mu_{a t}(e)}+R_{t}+(\log 2) N(1 / t)<\frac{(1+\delta)(\log 4 a)}{2(1+\delta / 2)} N(1 / t) .
$$

Hence, for $t \in\left(0, t_{0}\right)$, we have

$$
\mathbb{P}_{e}\left(d\left(X_{t}\right)>r\right) \leqslant(1+\delta) \exp \left(\frac{1+\delta}{2(1+\delta / 2)}(\log 4 a) N(1 / t)-\frac{r^{2}}{4(1+\delta) t}\right) .
$$

Let

$$
A=A(\delta)=\frac{4(1+\delta)^{2}(1+2 \delta) \log 4 a}{\delta(2+\delta)} .
$$

Then, for $r^{2} \geqslant \operatorname{AtN}(1 / t)$, we have

$$
\mathbb{P}_{e}\left(d\left(X_{t}\right)>r\right) \leqslant(1+\delta) \exp \left(-\frac{r^{2}}{4(1+2 \delta) t}\right) .
$$

Replacing $\delta$ by $\delta / 2$ gives (6.1) with

$$
C_{0}(\delta)=\frac{(2+\delta)^{2}(1+\delta) \log (4+8 / \delta)}{\delta(1+\delta / 4)} .
$$

Finnaly, since $\delta^{2} C_{0}(\delta)$ is increasing on $(0,1]$, we have $C_{0}(\delta) \leqslant C_{0}(1) \delta^{-2} \leqslant 36 \delta^{-2}$ as desired.

As $N \in \mathcal{R}_{0}$, the function $s N(1 / s)$ is equivalent to an increasing function as $s$ tends to 0 . Thus there exists $t_{0}^{\prime}=t_{0}^{\prime}(\delta)$ such that, for all $t \in\left(0, t_{0}^{\prime}\right)$ and all $r^{2} \geqslant \widetilde{C}_{0} t N(1 / t)$,

$$
\sup _{s \in(0, t)}\left\{\mathbb{P}_{e}\left(d\left(X_{s}\right)>r\right)\right\} \leqslant(1+\delta / 2) \exp \left(-\frac{r^{2}}{4(1+\delta) t}\right),
$$

where $\widetilde{C}_{0}(\delta)=(5 / 4) C_{0}(\delta)$ (the factor of $5 / 4$ accounts for the fact that $s N(1 / s)$ is not necessarily increasing but is equivalent to an increasing function).

Next, set $\tau=\inf \left\{s>0: d\left(X_{s}\right) \geqslant r\right\}$. By the strong Markov property, for any $\varepsilon \in(0,1)$, we have

$$
\mathbb{P}_{e}\left(d\left(X_{t}\right) \geqslant \varepsilon r\right) \geqslant \mathbb{P}_{e}(\tau \leqslant t)\left(1-\sup _{s \in(0, t)} \mathbb{P}_{e}\left(d\left(X_{s}\right) \geqslant(1-\varepsilon) r\right)\right)
$$

(see, e.g., [7]). Let $\varepsilon=\varepsilon(\delta)$ be given by

$$
\varepsilon^{2}=(1+\delta) /(1+2 \delta) \text {. }
$$

Then $\varepsilon^{2}>1 / 2$ and $(1-\varepsilon)^{2}<1 / 2$. Therefore if we set $A_{1}=A_{1}(\delta)=\widetilde{C}_{0}(1-\varepsilon)^{-2}$, for any $r$ such that $r^{2} \geqslant A_{1} t N(1 / t)$ both $(\varepsilon r)^{2}$ and $[(1-\varepsilon) r]^{2}$ are greater than $\widetilde{C}_{0} t N(1 / t)$. Hence $(6.3)$ yields

$$
\mathbb{P}_{e}(\tau \leqslant t) \leqslant \frac{(1+\delta / 2) \exp \left(-\frac{\varepsilon^{2} r^{2}}{4(1+\delta) t}\right)}{1-(1+\delta / 2) \exp \left(-\frac{(1-\varepsilon)^{2} r^{2}}{4(1+\delta) t}\right)} \leqslant \frac{(1+\delta / 2) \exp \left(-\frac{r^{2}}{4(1+2 \delta) t}\right)}{1-(1+\delta / 2) \exp \left(-\frac{\widetilde{C}_{0}}{4(1+\delta)} N(1 / t)\right)} .
$$


Finally, if $t_{1}=t_{1}(\delta) \leqslant t_{0}^{\prime}$ is chosen so small that for all $0<t<t_{1}$ we have

$$
1-(1+\delta / 2) \exp \left(-\frac{\widetilde{C}_{0}}{4(1+\delta)} N(1 / t)\right) \geqslant \frac{1}{2},
$$

then, for all $r, t>0$ with $0<t<t_{1}$ and $r^{2} \geqslant A_{1} t N(1 / t)$, we obtain

$$
\mathbb{P}_{e}(\tau \leqslant t) \leqslant(2+\delta) \exp \left(-\frac{r^{2}}{4(1+2 \delta) t}\right) .
$$

This gives the desired result with

$$
C_{1}(\delta)=(1-\varepsilon(\delta / 2))^{-2} \widetilde{C}_{0}(\delta / 2) \leqslant \frac{20(1+\delta)(2+3 \delta / 2)(2+\delta / 2)^{2}(1+\delta / 2) \log (4+16 / \delta)}{\delta^{3}(1+\delta / 8)} .
$$

For $\delta \in(0,1]$, we have $C_{1}(\delta) \leqslant 2^{12} \delta^{-4}$ as desired.

\subsection{Lévy-Khintchine-type upper bounds}

Theorem 6.2. Let $\mathbf{a}=\left(a_{i}\right)_{1}^{\infty}, a_{i}>0$ be such that $N=N^{\mathbf{a}}$ is slowly varying.

(1) If $N(s) \geqslant \eta \log \log s$ at infinity with $\eta \in(0,1)$ then, $\mathbb{P}_{e}$ almost surely,

$$
\limsup _{t \rightarrow 0} \frac{d\left(X_{t}\right)}{\sqrt{t N(1 / t)}} \leqslant \max \left\{2^{6}, 2^{3 / 2} \eta^{-1 / 2}\right\} \text {. }
$$

(2) If $N(s) \leqslant \theta \log \log s$ at infinity with $\theta \in(0,1)$ then, $\mathbb{P}_{e}$ almost surely,

$$
1 \leqslant \limsup _{t \rightarrow 0} \frac{d\left(X_{t}\right)}{\sqrt{4 t \log \log 1 / t}} \leqslant D(\theta),
$$

where $D(\theta) \leqslant 2\left(1+2^{8} \theta\right)^{1 / 2}$ if $\theta \in(0,1]$ and $D(\theta) \leqslant \sqrt{1+8 \theta^{1 / 4}}$ for $\theta \in\left(0,2^{-10}\right)$.

(3) If $N(s)=\mathrm{o}(\log \log s)$ at infinity then, $\mathbb{P}_{e}$ almost surely,

$$
\limsup _{t \rightarrow 0} \frac{d\left(X_{t}\right)}{\sqrt{4 t \log \log 1 / t}}=1 \text {. }
$$

Proof. The lower bound

$$
1 \leqslant \limsup _{t \rightarrow 0} \frac{d\left(X_{t}\right)}{\sqrt{4 t \log \log 1 / t}}
$$

follows from the classical finite dimensional result (see, e.g., [8]) by projection.

For the upper bound, fix $\sigma \in(0,1), \delta>0$ and consider two cases: In case 1 , we assume that $N(s) \geqslant \eta \log \log s$ whereas, in case 2 , we assume that $N(s) \leqslant \theta \log \log s$. In both cases, we consider the events

$$
A_{i}=\left\{\sup _{t \in\left(0, \sigma^{i}\right)} d\left(X_{t}\right) \geqslant \sqrt{\sigma^{i} g\left(\sigma^{i}\right)}\right\}
$$

with

$$
g(t)= \begin{cases}2^{12} k N(1 / t) & \text { in case } 1, \\ 4 k \log \log 1 / t & \text { in case 2, }\end{cases}
$$

where $k \geqslant 1$ will be fixed later. For $\delta>0$, let $C_{1}(\delta)$ and $t_{0}=t_{0}(\delta)$ be as in Lemma 6.1. Let $i_{0}$ be the integer such that $\sigma^{i_{0}} \leqslant t_{0}$. Then Lemma 6.1 yields

$$
\mathbb{P}_{e}\left(A_{i}\right) \leqslant 4 \exp \left(-\frac{g\left(\sigma^{i}\right)}{4(1+\delta)}\right)
$$


for all $i \geqslant i_{0}$ and all $k>1, \delta>0$, such that

$$
\forall t \in\left(0, t_{0}\right), \quad g(t) \geqslant C_{1}(\delta) N(1 / t) \text {. }
$$

If $N(s) \geqslant \eta \log \log s$ at infinity, then (6.4) holds true for $\delta=1$ and any $k \geqslant 1$ since $C_{1}(1)<2^{12}$. In this case, for all $k>\max \left\{1,2^{-9} \eta^{-1}\right\}$, the series $\sum \mathbb{P}_{e}\left(A_{i}\right)$ converges. Hence, by the Borel-Cantelli lemma, $\mathbb{P}_{e}$ almost surely, for all $n$ large enough,

$$
\sup _{t \in\left(0, \sigma^{n}\right)} d\left(X_{t}\right) \leqslant \sqrt{2^{12} k \sigma^{n} N\left(\sigma^{-n}\right)}
$$

As $t N(1 / t)$ is equivalent to an increasing function near 0 and $N(1 / t)$ is decreasing, it follows that $\mathbb{P}_{e}$ almost surely, for all $\varepsilon>0$ and for all $t$ small enough (depending on $\varepsilon$ ),

$$
d\left(X_{t}\right) \leqslant \sqrt{2^{12} k(1+\varepsilon)(t / \sigma) N(\sigma / t)} \leqslant \sqrt{2^{12} k(1+\varepsilon) \sigma^{-1} t N(1 / t)}
$$

Hence, $\mathbb{P}_{e}$ almost surely,

$$
\limsup _{t \rightarrow 0} \frac{d\left(X_{t}\right)}{\sqrt{t N(1 / t)}} \leqslant 2^{6}[(1+\varepsilon) k]^{1 / 2} \sigma^{-1 / 2} .
$$

As $\sigma \in(0,1), k>\max \left\{1,2^{-9} \eta^{-1}\right\}$ and $\varepsilon>0$ are arbitrary, this proves the first statement of Theorem 6.2.

Assume now that $N(s) \leqslant \theta \log \log s$ at infinity with $\theta \in(0,1]$. Let $k>1$ and $\delta \in(0,1)$ be such that

$$
4 k \geqslant \theta C_{1}(\delta) \text { and } k>1+\delta .
$$

Then (6.4) holds. It follows that

$$
\mathbb{P}_{e}\left(A_{i}\right) \leqslant 4 \exp \left(-\frac{k}{1+\delta} \log \log \sigma^{-i}\right)
$$

and that the series $\sum \mathbb{P}_{e}\left(A_{i}\right)$ converges. Applying the same line of reasoning as above, we find that $\mathbb{P}_{e}$ almost surely,

$$
\limsup _{t \rightarrow 0} \frac{d\left(X_{t}\right)}{\sqrt{4 t \log \log 1 / t}} \leqslant \sqrt{k} .
$$

The pair $k=4\left(1+2^{8} \theta\right), \delta=1$ satisfy (6.5) for all $\theta \in(0,1]$. To obtain a better bound when $\theta$ is smaller than $2^{-10}$, define $\delta(\theta)$ by

$$
C_{1}(\delta)=4 / \theta
$$

and pick any $k>1+\delta$. Then (6.5) is satisfied and we obtain

$$
\limsup _{t \rightarrow 0} \frac{d\left(X_{t}\right)}{\sqrt{4 t \log \log 1 / t}} \leqslant \sqrt{1+\delta(\theta)} \text {. }
$$

Moreover, the estimate $C_{1}(\delta) \leqslant 2^{12} \delta^{-4}$ in Lemma 6.1 implies that $\delta(\theta) \leqslant 8 \theta^{1 / 4}$. This finishes proof of the second statement of Theorem 6.2.

The third statement follows from the second statement by letting $\theta$ tend to 0 .

\subsection{Lévy's modulus of continuity: upper bounds}

Theorem 6.3. Let $\mathbf{a}=\left(a_{i}\right)_{1}^{\infty}, a_{i}>0$ be such that $N=N^{\mathbf{a}}$ is slowly varying.

(1) If $N(\tau) \geqslant \eta \log \tau$ at infinity with $\eta \in(0,1)$ then, $\mathbb{P}_{e}$ almost surely,

$$
\lim _{\varepsilon \rightarrow 0} \sup _{\substack{0<s<t<1 \\ t-s<\varepsilon}} \frac{d\left(X_{s}, X_{t}\right)}{\sqrt{(t-s) N(1 /(t-s))}} \leqslant \max \left\{8,5 \eta^{-1 / 2}\right\} .
$$


(2) If $N(\tau) \leqslant \theta \log \tau$ at infinity with $\theta \in(0,1)$ then, $\mathbb{P}_{e}$ almost surely,

$$
1 \leqslant \lim _{\varepsilon \rightarrow 0} \sup _{\substack{0<s<t<1 \\ t-s<\varepsilon}} \frac{d\left(X_{s}, X_{t}\right)}{\sqrt{4(t-s) \log 1 /(t-s)}}<D^{\prime}(\theta),
$$

where $D^{\prime}(\theta) \leqslant 8 \theta^{1 / 2}$ if $\theta>2 / 25$ and $D^{\prime}(\theta) \leqslant\left(1+6 \theta^{1 / 2}\right)^{2}$ for $\theta \in(0,2 / 25]$.

(3) If $N(\tau)=\mathrm{o}(\log \tau)$ at infinity then, $\mathbb{P}_{e}$ almost surely,

$$
\lim _{\varepsilon \rightarrow 0} \sup _{\substack{0<s<t<1 \\ t-s<\varepsilon}} \frac{d\left(X_{s}, X_{t}\right)}{\sqrt{4(t-s) \log 1 /(t-s)}}=1 .
$$

Proof. As was the case for Theorem 6.2, the lower bound follows from the classical finite dimensional result (see, e.g., [8]) by projection.

For the upper bounds, we again consider two cases: In case 1, we assume that $N(s) \geqslant \eta \log s$ whereas, in case 2 , we assume that $N(s) \leqslant \theta \log s$. For $t \in(0,1)$, we set

$$
g(t)= \begin{cases}50 k N(1 / t) & \text { in case } 1, \\ k \log 1 / t & \text { in case } 2,\end{cases}
$$

where $k>0$ will be chosen later. Set also

$$
h(t)=4 \operatorname{tg}(t) .
$$

Note that $g$ is non-increasing and slowly varying whereas $h$ is regularly varying of index 1 .

Let $\delta \in(0,1)$ be fixed and set

$$
K_{n}=\left\{(i, j) \in \mathbb{N}^{2}: 0 \leqslant i<j \leqslant 2^{n}, j-i \leqslant 2^{\delta n}\right\} .
$$

It is clear that $\# K_{n} \leqslant 2^{(1+\delta) n}$, and for $(i, j) \in K_{n}$, we have $(j-i) 2^{-n} \leqslant 2^{-n(1-\delta)}$. Consider the events

$$
A_{n}=\left\{\sup _{(i, j) \in K_{n}} \frac{d\left(X_{i 2^{-n}}, X_{j 2^{-n}}\right)}{\sqrt{h\left((j-i) 2^{-n}\right)}} \geqslant 1\right\} .
$$

We have

$$
\mathbb{P}_{e}\left(A_{n}\right) \leqslant \sum_{(i, j) \in K_{n}} \mathbb{P}_{e}\left(\frac{d\left(X_{i 2^{-n}}, X_{j 2^{-n}}\right)}{\sqrt{h\left((j-i) 2^{-n}\right)}} \geqslant 1\right) .
$$

Assume that $k>0$ and $\delta \in(0,1)$ are such that

$$
\limsup _{t \rightarrow 0} \frac{N(1 / t)}{g(t)}<\frac{4}{C_{0}(\delta)}
$$

$\left(C_{0}(\delta)\right.$ as in Lemma 6.1) and

$$
\sum_{n} 2^{(1+\delta) n} \mathrm{e}^{-(1+\delta)^{-1} g\left(2^{-(1-\delta) n}\right)}<\infty .
$$

We will see later how to find pairs $k, \delta$ satisfying both (6.6) and (6.7).

Let $n_{0}=n_{0}(k, \delta)$ be an integer such that

$$
\sup _{t \leqslant 2^{-n} 0^{(1-\delta)}} \frac{N(1 / t)}{g(t)} \leqslant \frac{4}{C_{0}(\delta)} .
$$

Then, for any such pair $k, \delta$ and all $n \geqslant n_{0}$, Lemma 6.1 gives 


$$
\begin{aligned}
\mathbb{P}_{e}\left(A_{n}\right) & \leqslant 2 \sum_{(i, j) \in K_{n}} \exp \left(-\frac{h\left((j-i) 2^{-n}\right)}{4(1+\delta)(j-i) 2^{-n}}\right) \\
& =2 \sum_{(i, j) \in K_{n}} \exp \left(-(1+\delta)^{-1} g\left((j-i) 2^{-n}\right)\right) \\
& \leqslant 2^{1+(1+\delta) n} \exp \left(-(1+\delta)^{-1} g\left(2^{-(1-\delta) n}\right)\right)
\end{aligned}
$$

For the last inequality, we have used that $g$ is non-increasing. This and (6.7) shows that $\sum \mathbb{P}_{e}\left(A_{n}\right)<\infty$. Hence the Borel-Cantelli lemma implies that, for $\mathbb{P}_{e}$ almost all $\omega \in \Omega$, there exists an integer $m(\omega)$ such that for all $n \geqslant m(\omega)$ and for all $(i, j) \in K_{n}$,

$$
d\left(X_{i 2^{-n}}, X_{j 2^{-n}}\right) \leqslant \sqrt{h\left((j-i) 2^{-n}\right)}
$$

Fix $\omega \in \Omega$ such that (6.8) holds. Fix $0 \leqslant s<t \leqslant 1$ with $0<t-s<2^{-(1-\delta) m(\omega)}$ and let $n \geqslant m(\omega)$ be such that $2^{-(1-\delta)(n+1)} \leqslant t-s<2^{-(1-\delta) n}$. Let $i$ be the smallest integer such that $s \leqslant i 2^{-n}$ and $j$ be the largest integer such that $j 2^{-n} \leqslant t$. Then we have $i<j$ provided that $2^{-(1-\delta)(n+1)} \geqslant 2^{-n+2}$. This is certainly satisfied if $n$ is greater than $3 \delta^{-1}$, which we can assume without loss of generality. Under this condition, we have

$$
0<j-i \leqslant 2^{n}(t-s) \leqslant 2^{\delta n}
$$

and thus, $(i, j) \in K_{n}$. It follows that (6.8) applies. In particular, since $h$ is equivalent to an increasing function at 0 , for any $\tau>0$ and $n$ large enough (depending on $\tau$ ) we get

$$
\begin{aligned}
& d\left(X_{i 2^{-n}}, X_{j 2^{-n}}\right) \leqslant(1+\tau) \sqrt{h(t-s)}, \\
& s=i 2^{-n}-2^{-u_{1}}-2^{-u_{2}}-\cdots, \\
& t=j 2^{-n}+2^{-v_{1}}+2^{-v_{2}}+\cdots,
\end{aligned}
$$

where $\left(u_{i}\right),\left(v_{i}\right)$ are increasing sequences of integers greater than $n$. Observe that, for each $\ell$, the pairs

$$
\begin{aligned}
& \left(i 2^{-n}-2^{-u_{1}}-\cdots-2^{-u_{\ell+1}}, i 2^{-n}-2^{-u_{1}}-\cdots-2^{-u_{\ell}}\right), \\
& \left(j 2^{-n}+2^{-v_{1}}+\cdots+2^{-v_{\ell}}, i 2^{-n}+2^{-v_{1}}+\cdots+2^{-v_{\ell+1}}\right)
\end{aligned}
$$

are in $K_{n}$. Using (6.8) and the fact that $t \mapsto X_{t}(\omega)$ is continuous, we obtain

$$
d\left(X_{s}, X_{i 2^{-n}}\right) \leqslant \sum_{\ell>n} \sqrt{h\left(2^{-\ell}\right)} .
$$

As $h$ is regularly varying of index 1 , for any $\eta>0$ there exists $C_{\eta}$ such that for all $n<\ell$ large enough,

$$
\frac{h\left(2^{-\ell}\right)}{h\left(2^{-n}\right)} \leqslant C_{\eta} 2^{(1-\eta)(n-\ell)} .
$$

Hence

$$
\begin{aligned}
d\left(X_{s}, X_{i 2^{-n}}\right) & \leqslant 2 C_{\eta} \sqrt{h\left(2^{-n}\right)} \sum_{\alpha \geqslant 1} 2^{-\frac{1}{2}(1-\eta) \alpha} \leqslant 2 C_{\eta}^{\prime} \sqrt{h\left(2^{-n}\right)} \\
& \leqslant \frac{2 C_{\eta}^{\prime} \sqrt{h\left(2^{-n}\right)}}{\sqrt{h\left(2^{-(1-\delta)(n+1)}\right)}} \sqrt{h\left(2^{-(1-\delta)(n+1)}\right)} \leqslant C_{\eta}^{\prime \prime} 2^{(1-\eta)(1-\delta(n+1)) / 2} \sqrt{h(t-s)}
\end{aligned}
$$

for all $n$ large enough. The same argument yields the same upper bound for $d\left(X_{j 2^{-n}}, X_{t}\right)$. Hence

$$
d\left(X_{s}, X_{t}\right) \leqslant\left(1+\tau+2 C_{\eta}^{\prime \prime} 2^{(1-\eta)(1-\delta(n+1)) / 2}\right) \sqrt{h(t-s)}
$$


It follows that

$$
\lim _{\varepsilon \rightarrow 0} \sup _{\substack{0<s<t<1 \\ t-s<\varepsilon}} \frac{d\left(X_{s}, X_{t}\right)}{\sqrt{h(t-s)}} \leqslant 1 .
$$

Here we have used $\tau>0$ can be picked arbitrary small.

We are left with the task to check that we can choose pairs $k, \delta$ satisfying (6.6) and (6.7) and such that the last inequality gives the desired results.

Consider the case where $N(s) \geqslant \eta \log s$ at infinity. Note that the constant $C_{0}(\delta)$ of Lemma 6.1 satisfies $C_{0}(1 / 2)<50$. Hence, for any $k \geqslant 1 / 4$, the pair $k, \delta=1 / 2$ satisfies (6.6). Moreover, in this case

$$
\begin{aligned}
\sum_{n} 2^{(1+\delta) n} \mathrm{e}^{-(1+\delta)^{-1} g\left(2^{(1-\delta) n}\right)} & =\sum_{n} 2^{(3 / 2) n} \mathrm{e}^{-(100 / 3) k N\left(2^{n / 2}\right)} \\
& \leqslant \sum_{n} 2^{(3 / 2) n} \mathrm{e}^{-(100 / 3) \eta k \log \left(2^{n / 2}\right)} \\
& =\sum_{n} \mathrm{e}^{-(\log 2)[(50 \eta k / 3)-(3 / 2)] n}
\end{aligned}
$$

Hence (6.7) is satisfied if $k>\max \left\{1 / 4,9(100 \eta)^{-1}\right\}$. Applying (6.10) for such $k$ and $\delta=1 / 2$ gives

$$
\lim _{\varepsilon \rightarrow 0} \sup _{\substack{0<s<t<1 \\ t-s<\varepsilon}} \frac{d\left(X_{s}, X_{t}\right)}{\sqrt{(t-s) N(t-s)}} \leqslant \max \left\{8,5 \eta^{-1 / 2}\right\}
$$

as stated in Theorem 6.3(1).

Next we consider the case where $N(s) \leqslant \theta \log s$ at infinity, with $\theta \in(0,1]$. Assume first that $\theta>2 / 25$. Observe that (6.6) is satisfied for all $k \geqslant 25 \theta / 2$ and $\delta=1 / 2$. Moreover, if $k>3^{2} 5^{2} \theta / 4$,

$$
\sum_{n} 2^{(1+\delta) n} \mathrm{e}^{-(1+\delta)^{-1} g\left(2^{(1-\delta) n}\right)}=\sum_{n} \mathrm{e}^{-(\log 2)[(k / 3)-(3 / 2)] n}<\infty,
$$

that is (6.7) is also satisfied. Hence (6.10) yields

$$
\lim _{\varepsilon \rightarrow 0} \sup _{\substack{0<s<t<1 \\ t-s<\varepsilon}} \frac{d\left(X_{s}, X_{t}\right)}{\sqrt{4(t-s) \log (1 / t-s)}} \leqslant 8 \theta^{1 / 2}
$$

as desired. If $\theta \in(0,2 / 25]$, we instead define $\delta=\delta(\theta) \in(0,1 / 2)$ by

$$
4 / \theta=C_{0}(\delta) .
$$

For this $\delta,(6.6)$ is satisfied for all $k>1$. For $k=(1+\delta)^{3}(1-\delta)^{-1}$, we have

$$
\sum_{n} 2^{(1+\delta) n} \mathrm{e}^{-(1+\delta)^{-1} g\left(2^{(1-\delta) n}\right)}=\sum_{n} 2^{(1+\delta) n-(1+\delta)^{2} n}=\sum_{n} 2^{-\delta(1+\delta) n}
$$

and (6.7) is also satisfied. Thus (6.10) applies and gives

$$
\lim _{\varepsilon \rightarrow 0} \sup _{\substack{0<s<t<1 \\ t-s<\varepsilon}} \frac{d\left(X_{s}, X_{t}\right)}{\sqrt{4(t-s) \log (1 / t-s)}} \leqslant \sqrt{\frac{(1+\delta)^{3}}{1-\delta}} .
$$

The estimate $C_{0}(\delta) \leqslant 36 \delta^{-2}$ in Lemma 6.1 and equation $C_{0}(\delta)=4 / \theta$ gives $\delta \leqslant 3 \theta^{1 / 2}$. As $(1-\delta)^{-1} \leqslant 1+2 \delta$ when $\delta \in(0,1 / 2)$, we obtain

$$
\lim _{\varepsilon \rightarrow 0} \sup _{\substack{0<s<t<1 \\ t-s<\varepsilon}} \frac{d\left(X_{s}, X_{t}\right)}{\sqrt{4(t-s) \log (1 / t-s)}} \leqslant\left(1+6 \theta^{1 / 2}\right)^{2}
$$


as announced in Theorem 6.3.

Finally, the last statement of Theorem 6.3 follows from the last inequality by letting $\theta$ tend to 0 .

\section{Regularity of paths: lower bounds}

This section is devoted to proving lower bounds that complement the upper bounds proved in Sections 6.2 and 6.3.

\subsection{Lévy-Khinchine-type lower bound}

Theorem 7.1. Let $\mathbf{a}=\left(a_{i}\right)_{1}^{\infty}, a_{i}>0$, be such that $N=N^{\mathbf{a}}$ is slowly varying. Then, $\mathbb{P}_{e}$ almost surely,

$$
\limsup _{t \rightarrow 0} \frac{d\left(X_{t}\right)}{\sqrt{t N(1 / t)}} \geqslant(2 / \mathrm{e})^{1 / 2} .
$$

Remark. If $N(s) \leqslant 2 \mathrm{e} \log \log s$ at infinity, then the lower bound stated above is weaker than the lower bound

$$
\limsup _{t \rightarrow 0} \frac{d\left(X_{t}\right)}{\sqrt{4 t \log \log (1 / t)}} \geqslant 1
$$

which follows by projection from the classical finite dimensional law of the iterated logarithm. If $N(t) \geqslant$ $8 \mathrm{e}(1+\eta) \log \log s$ then the lower bound of Theorem 7.1 shows that

$$
\limsup _{t \rightarrow 0} \frac{d\left(X_{t}\right)}{\sqrt{4 t \log \log (1 / t)}} \geqslant(1+\eta)^{1 / 2} .
$$

Hence, in this case, the classical law of the iterated logarithm does not hold.

Proof of Theorem 7.1. Fix a sequence $t_{n}$ tending to zero and consider the event

$$
A_{n}=\left\{d\left(X_{t_{n}}\right) \leqslant h\left(t_{n}\right)\right\},
$$

where $h(t)=\sqrt{4 k t N(1 / t)}$ and $k$ will be chosen later. We have

$$
\mathbb{P}_{e}\left(A_{n}\right) \leqslant \mu_{t_{n}}(e) V\left(h\left(t_{n}\right)\right) \text {. }
$$

Using Proposition 5.4 and the definition of $h$, for any $\theta \in(0,1)$, we have

$$
V(h(t)) \leqslant \frac{\mathrm{e}^{(k / \theta) N(1 / t)}}{\mu_{\theta t}(e)} .
$$

Hence,

$$
\mu_{t}(e) V(h(t)) \leqslant \frac{\mu_{t}(e)}{\mu_{\theta t}(e)} \exp \left(\frac{k}{\theta} N(1 / t)\right) .
$$

By Proposition 3.3, this gives

$$
\mu_{t}(e) V(h(t)) \leqslant \exp \left(\frac{1-\varepsilon}{2}(\log \theta) N(1 / t)+\frac{k}{\theta} N(1 / t)\right)
$$

for any $\varepsilon \in(0,1)$ and $t$ small enough. We pick $\theta=1 / \mathrm{e}$ and $k=(1-2 \varepsilon) / 2 \mathrm{e}$ with $\varepsilon \in(0,1 / 2)$ to obtain

$$
\mu_{t}(e) V(h(t)) \leqslant \exp \left(-\frac{\varepsilon}{2} N(1 / t)\right) .
$$


Now, we choose the sequence $t_{n}$ so that $N\left(1 / t_{n}\right) \geqslant n$. It follows that $\sum_{n} \mathbb{P}_{e}\left(A_{n}\right)$ converges. By the Borel-Cantelli lemma, $\mathbb{P}_{e}$ almost surely, for all $n$ large enough,

$$
d\left(X_{t_{n}}\right)>h\left(t_{n}\right) \text {. }
$$

Hence

$$
\limsup _{t \rightarrow 0} \frac{d\left(X_{t}\right)}{\sqrt{t N(1 / t)}} \geqslant \sqrt{2(1-2 \varepsilon) / \mathrm{e}} .
$$

As $\varepsilon \in(0,1 / 2)$ is arbitrary, we obtain the desired result by letting $\varepsilon$ tend to 0 .

\subsection{Modulus of continuity: lower bound}

Theorem 7.2. Let $\mathbf{a}=\left(a_{i}\right)_{1}^{\infty}, a_{i}>0$, be such that $N=N^{\mathbf{a}}$ is slowly varying. Then, $\mathbb{P}_{e}$ almost surely,

$$
\lim _{\varepsilon \rightarrow 0} \sup _{\substack{0<s<t<1 \\ t-s<\varepsilon}} \frac{d\left(X_{s}, X_{t}\right)}{\sqrt{(t-s) N(1 /(t-s))}} \geqslant(2 / \mathrm{e})^{1 / 2} .
$$

Remark. If $N(s) \leqslant 2 e \log s$ at infinity, then this result is weaker than the inequality

$$
\lim _{\varepsilon \rightarrow 0} \sup _{\substack{0<s<t<1 \\ t-s<\varepsilon}} \frac{d\left(X_{s}, X_{t}\right)}{\sqrt{4(t-s) \log (1 /(t-s))}} \geqslant 1
$$

obtained by looking at any finite dimensional projection. If $N(s) \geqslant 8 e(1+\eta) \log s$ at infinity, then Theorem 7.2 shows that the classical result of Lévy

$$
\lim _{\varepsilon \rightarrow 0} \sup _{\substack{0<s<t<1 \\ t-s<\varepsilon}} \frac{d\left(X_{s}, X_{t}\right)}{\sqrt{4(t-s) \log (1 /(t-s))}}=1
$$

does not hold since, in this case, the left-hand side is at least $(1+\eta)^{1 / 2}$.

Proof of Theorem 7.2. Consider the event

$$
A_{n}=\left\{\sup _{0 \leqslant j<2^{n}} d\left(X_{j 2^{-n}}, X_{(j+1) 2^{-n}}\right) \leqslant h\left(2^{-n}\right)\right\},
$$

where $h(t)^{2}=4(1-2 \eta)(2 \mathrm{e})^{-1} t N(1 / t)$ with $\eta \in(0,1 / 2)$ arbitrary. Then, as the increments $\left(X_{j 2^{-n}}^{-1} X_{\left.(j+1) 2^{-n}\right) \text { are }}\right.$ independent and identically distributed, we have

$$
\mathbb{P}_{e}\left(A_{n}\right)=\left(\mathbb{P}_{e}\left(d\left(X_{2^{-n}}\right) \leqslant h\left(2^{-n}\right)\right)\right)^{2^{n}}
$$

Using (7.12), we obtain

$$
\mathbb{P}_{e}\left(A_{n}\right) \leqslant \exp \left(-\eta 2^{n-1} N\left(2^{n}\right)\right)
$$

for any $\eta \in(0,1 / 2)$ and $n$ large enough. This implies that the series $\sum_{n} \mathbb{P}_{e}\left(A_{n}\right)$ converges. By the Borel-Cantelli lemma, $\mathbb{P}_{e}$ almost surely,

$$
\sup _{0 \leqslant j \leqslant 2^{n}} d\left(X_{j 2^{-n}}, X_{(j+1) 2^{-n}}\right) \geqslant h\left(2^{-n}\right),
$$

for all $n$ large enough. Hence

$$
\lim _{\varepsilon \rightarrow 0} \sup _{\substack{0 \leqslant s<t \leqslant 1 \\ t-s<\varepsilon}} \frac{d\left(X_{s}, X_{t}\right)}{\sqrt{(t-s) N(1 /(t-s))}} \geqslant \sqrt{2(1-2 \eta) / \mathrm{e}} .
$$

As $\eta \in(0,1 / 2)$ is arbitrary, this gives the desired result. 


\subsection{Dvoretzky-Erdös-type results}

In [14], Dvoretzky and Erdös studied the rate of escape of the square lattice random walk in dimension greater or equal to 3 . From a local viewpoint, this amount to studying

$$
\liminf _{t \rightarrow 0} \frac{d\left(X_{t}\right)}{\psi(t)} \text {. }
$$

For Brownian motion on the finite dimensional torus of dimension $n \geqslant 3$, the Dvoretzky-Erdös-type result states that, for any increasing function $\phi$ we have (see, e.g., [8])

$$
\liminf _{t \rightarrow 0} \frac{d\left(X_{t}\right)}{\sqrt{t \phi(t)}}=\left\{\begin{array} { l } 
{ \infty } \\
{ 0 }
\end{array} \text { a.s. iff the series } \sum _ { n } \phi ( 2 ^ { - n } ) ^ { ( n - 2 ) / 2 } \left\{\begin{array}{l}
\text { converges, } \\
\text { diverges. }
\end{array}\right.\right.
$$

For instance, we have

$$
\liminf _{t \rightarrow 0} \frac{d\left(X_{t}\right)}{\sqrt{t(\log 1 / t)^{-\sigma}}}=\left\{\begin{array} { l } 
{ \infty } \\
{ 0 }
\end{array} \text { a.s. iff } \left\{\begin{array}{l}
\sigma>\frac{2}{n-2}, \\
\sigma \leqslant \frac{2}{n-2} .
\end{array}\right.\right.
$$

Returning to the infinite dimensional torus $\mathbb{T}^{\infty}$, consider a Brownian motion $\left(X_{t}\right)_{t} \geqslant 0$ whose intrinsic distance $d$ is finite and defines the topology of $\mathbb{T}^{\infty}$. Then, looking a finite dimensional projections, we find that

$$
\liminf _{t \rightarrow 0} \frac{d\left(X_{t}\right)}{\sqrt{t(\log 1 / t)^{-\sigma}}}=\infty
$$

$\mathbb{P}_{e}$ almost surely for any $\sigma>0$. In [7], the authors give a large class of examples where, in fact, $\mathbb{P}_{e}$ almost surely,

$$
0<\liminf _{t \rightarrow 0} \frac{d\left(X_{t}\right)}{t^{(1-\lambda) / 2}} \leqslant \limsup _{t \rightarrow 0} \frac{d\left(X_{t}\right)}{t^{(1-\lambda) / 2}}<\infty .
$$

Here we consider the case of diagonal Brownian motions $X^{\mathbf{a}}$ associated with sequences $a_{i}$ such that $N^{\mathbf{a}}$ is slowly varying.

Theorem 7.3. Let $\mathbf{a}=\left(a_{i}\right)_{1}^{\infty}, a_{i}>0$, be such that $N=N^{\mathbf{a}}$ is slowly varying.

(1) One always has that, $\mathbb{P}_{e}$ almost surely,

$$
\liminf _{t \rightarrow 0} \frac{d\left(X_{t}\right)}{\sqrt{t N(1 / t)}} \leqslant 6 \text {. }
$$

(2) Assume that $N(s) \geqslant \eta \log \log s$ at infinity for some $\eta \in(0,4)$. Then, $\mathbb{P}_{e}$ almost surely,

$$
\liminf _{t \rightarrow 0} \frac{d\left(X_{t}\right)}{\sqrt{t N(1 / t)}} \geqslant 2^{-1} \mathrm{e}^{-2 / \eta}
$$

Proof of (1). To prove this it suffices to find a sequence $t_{n}$ such that the series $\sum_{n} \mathbb{P}_{e}\left(A_{n}\right)$ converges where

$$
A_{n}=\left\{d\left(X_{t_{n}}\right) \geqslant \sqrt{36 t_{n} N\left(1 / t_{n}\right)}\right\} .
$$

By (6.1) with $\delta=1$, we have

$$
\mathbb{P}_{e}\left(A_{n}\right) \leqslant 2 \exp \left(-(9 / 2) N\left(1 / t_{n}\right)\right) .
$$

Hence it suffices to pick $t_{n}$ such that $N\left(1 / t_{n}\right)=n$.

Proof of (2). Consider the process $X^{1}=\left(X_{t}^{1}\right)$ associated with the convolution semigroup $\left(\mathrm{e}^{-t} \mu_{t}^{\mathbf{a}}\right)_{t>0}$. This process takes value in $\mathbb{T}^{\infty} \cup\{\infty\}$ where $\infty$ is an isolated point added to $\mathbb{T}^{\infty}$. Set $d(x, \infty)=+\infty$ for any $x \in \mathbb{T}^{\infty}$. The 
process $X^{1}$ can be obtained from $X$ in the following way. Let $\xi$ be a real random variable, independent of the process $X$ and with $\mathbb{P}(\xi>t)=\mathrm{e}^{-t}$. Then

$$
X_{t}^{1}= \begin{cases}X_{t} & \text { if } t<\xi \\ \infty & \text { if } t \geqslant \xi\end{cases}
$$

Thus $X_{t}^{1}$ is $X_{t}$ killed at an exponential time.

Following the line of reasoning used in [7, Section 4.3], to prove that $\mathbb{P}_{e}$ almost surely,

$$
\liminf _{t \rightarrow 0} \frac{d\left(X_{t}\right)}{\sqrt{t N(1 / t)}} \geqslant \sqrt{k}
$$

it suffices to show that the series $\sum_{n} \mathbb{P}_{e}\left(B_{n}\right)$ converges where

$$
B_{n}=\left\{d\left(X_{t}^{1}\right)<\sqrt{k a^{n} N\left(a^{-n}\right)} \text { for some } t \geqslant a^{n+1}\right\}
$$

for some fixed $a \in(0,1)$. By [8, Theorem 3.9], we have

$$
\mathbb{P}_{e}\left(B_{n}\right) \leqslant \operatorname{Cap}^{1}\left(\mathbf{B}\left(\sqrt{k a^{n} N\left(a^{-n}\right)}\right)\right) \int_{a^{n+1}}^{\infty} \mathrm{e}^{-t} \mu_{t}(e) \mathrm{d} t,
$$

where $\operatorname{Cap}^{1}(A)$ denote the 1-capacity of the set $A$ (see, e.g., $[8,12,21]$ ) and $\mathbf{B}(r)$ is the closed intrinsic ball of radius $r$ centred at the identity element. This type of estimate is also used in [16] for a simlar purpose but in a different setting. We will use a very general capacity inequality proved in [21] (see also [8]) which gives

$$
\begin{aligned}
\operatorname{Cap}^{1}(\mathbf{B}(r)) & \leqslant 2\left(\int_{r}^{2 r} \frac{s-r}{V(s)-V(r)} \mathrm{d} s\right)^{-1}+V(2 r) \\
& \leqslant V(2 r)\left(1+2\left(\int_{r}^{2 r}(s-r) \mathrm{d} s\right)^{-1}\right) \\
& =V(2 r)\left(1+\frac{4}{r^{2}}\right) .
\end{aligned}
$$

Next we estimate $\int_{t}^{\infty} \mathrm{e}^{-s} \mu_{s}(e) \mathrm{d} s$. First we observe that there exists $t_{0} \in(0,1)$ such that $t \mapsto t^{2} \mu_{t}(e)$ is decreasing on $\left(0, t_{0}\right)$. Indeed, using Proposition 3.3, for $0<t<t_{0}$ with $t_{0}$ small enough and $1 / 2<c<1$, we have

$$
\frac{t^{2} \mu_{t}(e)}{(c t)^{2} \mu_{c t}(e)} \leqslant \exp \left(2\left(\log c^{-1}\right)-\frac{1-\varepsilon}{2}\left(\log c^{-1}\right) N(1 / t)\right) \leqslant 1 .
$$

Hence

$$
\begin{aligned}
\int_{t}^{\infty} \mathrm{e}^{-s} \mu_{s}(e) \mathrm{d} s & =\int_{t}^{t_{0}} \mathrm{e}^{-s} \mu_{s}(e) \mathrm{d} s+\int_{t_{0}}^{\infty} \mathrm{e}^{-s} \mu_{s}(e) \mathrm{d} s \leqslant C+t^{2} \mu_{t}(e) \int_{t}^{t_{0}} \frac{\mathrm{d} s}{s^{2}} \\
& \leqslant C+t^{2}\left(\frac{1}{t}-\frac{1}{t_{0}}\right) \mu_{t}(e) \leqslant C+t \mu_{t}(e) .
\end{aligned}
$$

Using (7.14) and (7.15) in (7.13), we obtain the estimate 


$$
\begin{aligned}
\mathbb{P}\left(B_{n}\right) & \leqslant C^{\prime} \frac{V\left(2 \sqrt{k a^{n} N\left(a^{-n}\right)}\right)}{k a^{n} N\left(a^{-n}\right)} a^{n+1} \mu_{a^{n+1}}(e) \\
& \leqslant \frac{a C^{\prime}}{k N\left(a^{-n}\right)}\left[V\left(2 \sqrt{k a^{n} N\left(a^{-n}\right)}\right) \mu_{a^{n}}(e)\right] \frac{\mu_{a^{n+1}}(e)}{\mu_{a^{n}}(e)},
\end{aligned}
$$

for all $n$ small enough. By Proposition 3.3 and (7.11), for any $\theta, \varepsilon \in(0,1)$, we obtain

$$
\mathbb{P}\left(B_{n}\right) \leqslant \frac{a C^{\prime}}{k N\left(a^{-n}\right)} \exp \left(\left(\frac{1-\varepsilon}{2}(\log \theta)+\frac{k}{\theta}\right) N\left(a^{-n}\right)+\left(\log a^{-1}\right) N\left(a^{-n}\right)\right)
$$

for all $n$ small enough. For $\theta=4 k \leqslant 1 / e$, we get

$$
\mathbb{P}\left(B_{n}\right) \leqslant \frac{a C^{\prime}}{k N\left(a^{-n}\right)} \exp \left(-\frac{1-2 \varepsilon}{4}\left(\log \theta^{-1}\right) N\left(a^{-n}\right)+\left(\log a^{-1}\right) N\left(a^{-n}\right)\right) .
$$

Assuming that $N(s) \geqslant \eta \log \log s$ at infinity with $\eta \in(0,4)$, we can pick $4 k=e^{-(1+\gamma) \frac{4}{\eta}}$ for some fixed $\gamma>0$. For this choice the series $\sum_{n} \mathbb{P}_{e}\left(B_{n}\right)$ converges if we pick $\varepsilon$ small enough and $a \in(0,1)$ close enough to 1 . Hence, we obtain

$$
\liminf _{t \rightarrow 0} \frac{d\left(X_{t}\right)}{\sqrt{t N(1 / t)}} \geqslant 2^{-1} \mathrm{e}^{-2(1+\gamma) / \eta}
$$

Since $\gamma>0$ is arbitrary, we obtain the desired result.

\section{Appendix A}

In this appendix, we compute the constant $c_{\lambda}$ of Theorem 4.1. Recall that for $\lambda \in(0,1)$

$$
c_{\lambda}=\int_{0}^{\infty} s^{\lambda} \Phi(s) \mathrm{d} s
$$

with

$$
\Phi(t)=-\frac{\mathrm{d}}{\mathrm{d} t}\left(\frac{\phi(t)}{t}\right) \text { and } \phi(t)=\frac{1}{2 \pi} \int_{-\pi}^{\pi} x^{2} v_{t}(x) \mathrm{d} x,
$$

where $v_{t}(x)$ is given at (2.1). We first claim that

$$
\phi(s)=\frac{\pi^{2}}{3}+4 \sum_{1}^{\infty} \frac{(-1)^{n}}{n^{2}} \mathrm{e}^{-s n^{2}} .
$$

Indeed, using the Fourier series for $v_{t}(x)$, we have

$$
\phi(s)=\frac{1}{\pi} \int_{0}^{\pi}\left(1+2 \sum_{1}^{\infty}(\cos n x) \mathrm{e}^{-s n^{2}}\right) x^{2} \mathrm{~d} x .
$$

Hence

$$
\begin{aligned}
\phi(s)-\frac{\pi^{2}}{3} & =\sum_{1}^{\infty}\left(\frac{2}{\pi} \int_{0}^{\pi} x^{2} \cos n x \mathrm{~d} x\right) \mathrm{e}^{-s n^{2}} \\
& =\sum_{1}^{\infty}\left(\frac{2}{\pi n} \int_{0}^{\pi n} x^{2} \cos x \mathrm{~d} x\right) n^{-2} \mathrm{e}^{-s n^{2}}=4 \sum_{n \geqslant 1} \frac{(-1)^{n}}{n^{2}} \mathrm{e}^{-s n^{2}}
\end{aligned}
$$


as desired. To compute $c_{\lambda}=\int_{0}^{\infty} s^{\lambda} \Phi(s) \mathrm{d} s, \lambda \in(0,1)$, use integration by parts to obtain

$$
\int_{0}^{\infty} s^{\lambda} \Phi(s) \mathrm{d} s=\lambda \lim _{\varepsilon \rightarrow 0} \int_{\varepsilon}^{\infty} \frac{\phi(s)}{s^{2-\lambda}} \mathrm{d} s .
$$

By (A.1), we get

$$
\begin{aligned}
\int_{\varepsilon}^{\infty} \frac{\phi(s)}{s^{2-\lambda}} \mathrm{d} s & =\int_{\varepsilon}^{\infty}\left(\frac{\pi^{2}}{3}+4 \sum_{n \geqslant 1} \frac{(-1)^{n}}{n^{2}} \mathrm{e}^{-s n^{2}}\right) \frac{\mathrm{d} s}{s^{2-\lambda}} \\
& =-\frac{\pi^{2} \varepsilon^{\lambda-1}}{3(\lambda-1)}+4 \sum_{n \geqslant 1} \frac{(-1)^{n}}{n^{2}} \int_{\varepsilon}^{\infty} \mathrm{e}^{-s n^{2}} \frac{\mathrm{d} s}{s^{2-\lambda}} .
\end{aligned}
$$

Next, integration by parts gives

$$
\int_{\varepsilon}^{\infty} \mathrm{e}^{-s n^{2}} \frac{\mathrm{d} s}{s^{2-\lambda}}=n^{2-2 \lambda} \int_{\varepsilon n^{2}}^{\infty} \mathrm{e}^{-s} \frac{\mathrm{d} s}{s^{2-\lambda}}=\frac{\varepsilon^{\lambda-1} \mathrm{e}^{-\varepsilon n^{2}}}{1-\lambda}-\frac{n^{2-2 \lambda}}{1-\lambda} \int_{\varepsilon n^{2}}^{\infty} \mathrm{e}^{-s} \frac{\mathrm{d} s}{s^{1-\lambda}} .
$$

Hence

$$
\int_{\varepsilon}^{\infty} \frac{\phi(s)}{s^{2-\lambda}} \mathrm{d} s=\frac{4}{1-\lambda}\left(\frac{\pi^{2} \varepsilon^{\lambda-1}}{12}+\sum_{n \geqslant 1} \frac{(-1)^{n}}{n^{2}} \varepsilon^{\lambda-1} \mathrm{e}^{-\varepsilon n^{2}}-\sum_{n \geqslant 1} \frac{(-1)^{n}}{n^{2 \lambda}} \int_{\varepsilon n^{2}}^{\infty} \mathrm{e}^{-s} \frac{\mathrm{d} s}{s^{1-\lambda}}\right) .
$$

Now, by (A.1) and Lemma 4.2, we have

$$
\frac{\pi^{2} \varepsilon^{\lambda-1}}{12}+\sum_{n \geqslant 1} \frac{(-1)^{n}}{n^{2}} \varepsilon^{\lambda-1} \mathrm{e}^{-\varepsilon n^{2}}=\frac{\varepsilon^{\lambda-1}}{4} \phi(\varepsilon) \sim \frac{\varepsilon^{\lambda}}{8}
$$

as $\varepsilon$ goes to zero. Hence, this part does not contribute to (A.2) and we have

$$
\int_{0}^{\infty} s^{\lambda} \Phi(s) \mathrm{d} s=-\frac{4 \lambda}{1-\lambda} \lim _{\varepsilon \rightarrow 0} \sum_{n \geqslant 1} \frac{(-1)^{n}}{n^{2 \lambda}} \int_{\varepsilon n^{2}}^{\infty} \mathrm{e}^{-s} \frac{\mathrm{d} s}{s^{1-\lambda}} .
$$

The above series is an alternating series. One can check that the summation and limit can be interchanged. Hence

$$
c_{\lambda}=\int_{0}^{\infty} s^{\lambda} \Phi(s) \mathrm{d} s=-\frac{4 \lambda}{1-\lambda} \sum_{n \geqslant 1} \frac{(-1)^{n}}{n^{2 \lambda}} \int_{0}^{\infty} \mathrm{e}^{-s} \frac{\mathrm{d} s}{s^{1-\lambda}}=\frac{4 \lambda \Gamma(\lambda)}{1-\lambda} \sum_{n \geqslant 1} \frac{(-1)^{n+1}}{n^{2 \lambda}} .
$$

This is the desired formula for $c_{\lambda}$.

\section{References}

[1] A. Bendikov, Space-homogeneous continuous Markov processes on Abelian groups and harmonic structures, Russian Math. Surveys 29 (5) (1974) 215-216.

[2] A. Bendikov, Potential Theory on Infinite Dimensional Abelian Groups, De Gruyter, Berlin, 1995.

[3] A. Bendikov, Symmetric stable semigroups on the infinite dimensional torus, Expositiones Math. 13 (1995) 39-79.

[4] A. Bendikov, L. Saloff-Coste, On- and off-diagonal heat kernel behaviors on certain infinite dimensional local Dirichlet spaces, Amer. J. Math. 122 (2000) 1205-1263. 
[5] A. Bendikov, L. Saloff-Coste, Central Gaussian semigroups of measures with continuous density, J. Funct. Anal. 186 (2001) $206-268$.

[6] A. Bendikov, L. Saloff-Coste, Central Gaussian convolution semigroups on compact groups: a survey, Infinite Dimensional Analysis, Quantum Probability and Related Topics, in press.

[7] A. Bendikov, L. Saloff-Coste, On the sample paths of Brownian motions on compact infinite dimensional groups, Ann. Probab. 31 (2003) 1464-1493.

[8] A. Bendikov, L. Saloff-Coste, On the sample paths of subelliptic diffusions on manifolds, in preparation.

[9] C. Berg, Potential theory on the infinite dimensional torus, Inventiones Math. 32 (1976) 49-100.

[10] N.H. Bingham, C.M. Goldie, J.L. Teugels, Regular Variation. Encyclopedia of Mathematics and its Applications, Cambridge University Press.

[11] M. Biroli, U. Mosco, Formes de Dirichlet et estimations structurelles dans les milieux discontinus, C. R. Acad. Sci. Paris 313 (1991) 593-598.

[12] R. Blumenthal, R. Getoor, Markov Processes and Potential Theory, Academic Press, 1968.

[13] E.B. Davies, Heat Kernels and Spectral Theory, Cambridge University Press, 1989.

[14] A. Dvoretzky, P. Erdös, Some problems on random walk in space, in: Second Berkeley Symp., University of California Press, 1951, pp. 353-367.

[15] M. Fukushima, Y. Oshima, M. Takeda, Dirichlet Forms and Symmetric Markov Processes, De Gruyter, 1994.

[16] A. Grigor'yan, Escape rate of Brownian motion on Riemannian manifolds, Appl. Anal. 71 (1999) 63-89.

[17] H. Heyer, Probability Measures on Locally Compact Groups, in: Ergeb. Math. Grenzgeb., vol. 94, Springer, Berlin, 1977.

[18] K. Ito, P. McKean, Diffusion Processes and their Sample Paths, Springer, 1974.

[19] D. Revuz, M. Yor, Continuous Martingales and Brownian Motion, Second edition, Springer, 1994.

[20] K.-T. Sturm, On the geometry defined by Dirichlet forms, in: E. Bolthausen, et al. (Eds.), Seminar on Stochastic Processes, Random Fields and Applications, Ascona, in: Progress in Probability, vol. 36, Birkhäuser, 1995, pp. 231-242.

[21] K.-T. Sturm, Sharp estimates for capacities and applications to symmetric diffusions, Probab. Theory Related Fields 103 (1995) $73-89$. 